\title{
The exponential generalized log-logistic model: Bagdonavičius-Nikulin test for validation and non-Bayesian estimation methods
}

\author{
Mohamed Ibrahim ${ }^{1, a}$, Khaoula Aidi $^{b}$, Mir Masoom Ali $^{d}$, Haitham M. Yousof $^{c}$ \\ ${ }^{a}$ Department of Applied, Mathematical and Actuarial Statistics, Damietta University, Egypt; \\ ${ }^{b}$ Laboratory of Probability and Statistics LaPS, University Badji Mokhtar, Algeria; \\ ${ }^{d}$ Department of Mathematical Sciences, Ball State University, Muncie, USA; \\ ${ }^{c}$ Department of Statistics, Mathematics and Insurance, Benha University, Egypt
}

\begin{abstract}
A modified Bagdonavičius-Nikulin chi-square goodness-of-fit is defined and studied. The lymphoma data is analyzed using the modified goodness-of-fit test statistic. Different non-Bayesian estimation methods under complete samples schemes are considered, discussed and compared such as the maximum likelihood least square estimation method, the Cramer-von Mises estimation method, the weighted least square estimation method, the left tail-Anderson Darling estimation method and the right tail Anderson Darling estimation method. Numerical simulation studies are performed for comparing these estimation methods. The potentiality of the new model is illustrated using three real data sets and compared with many other well-known generalizations.
\end{abstract}

Keywords: Bagdonavičius-Nikulin test, Barzilai-Borwein, Anderson Darling estimation, log-logistic model, Cramér-von-Mises estimation

\section{Introduction and motivation}

In this paper, we extended the log-logistic (LL) model by proposing and studying a new probability distribution called the exponential generalized log-logistic (EG-LL) model. Different non-Bayesian well-known estimation methods under complete samples scheme are considered and discussed such as, the maximum likelihood estimation (MLE), the Anderson Darling estimation (ADE), the ordinary least square estimation (OLSE), the Cramér-von-Mises estimation (CVME), the weighted least square estimation (WLSE), the left tail-Anderson Darling estimation (ADE(L-T)), and the right tail Anderson Darling estimation (ADE(R-T)) methods. Numerical simulation studies are performed for comparing these estimation methods using different sample sizes and three different combinations of parameters. Some useful comments are made and recorded in Sections 4 and 5. The potentiality of the EGLL model is illustrated using three real data sets. The EG-LL model is compared with many other well-known generalizations. The new model proved its worth in modeling breaking stress, survival times and medical data sets. The Barzilai-Borwein (BB) algorithm is employed via a simulation study for assessing the performance of the estimators with different sample sizes as sample size tends to $\infty$. Using the Bagdonavičius-Nikulin goodness-of-fit test for validation, we propose a modified chi-square GOF tests for the EG-LL model. We have analyzed a lymphoma data set consisting of

\footnotetext{
${ }^{1}$ Corresponding author: Department of Applied, Mathematical and Actuarial Statistics, Damietta University, Egypt.

E-mail: mohamed_ibrahim@du.edu.eg
}

Published 31 January 2022 / journal homepage: http://csam.or.kr

(c) 2022 The Korean Statistical Society, and Korean International Statistical Society. All rights reserved. 

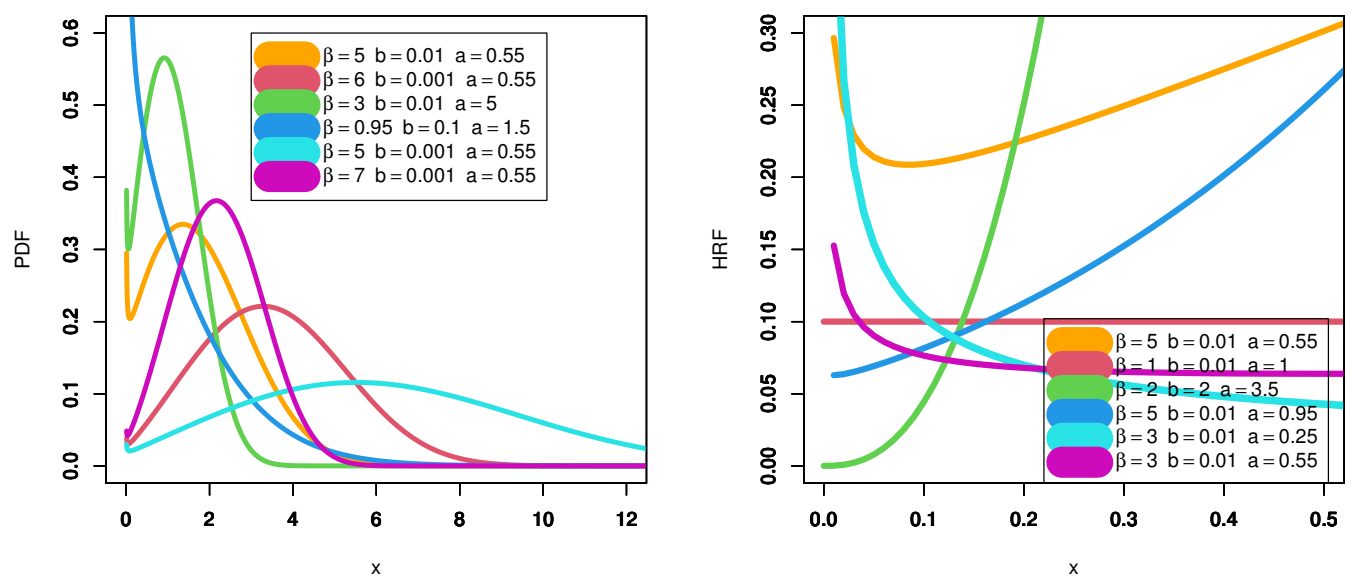

Figure 1: Plots for the PDF and HRF for the EG-LL model.

times (in months) from diagnosis to death for 31 individuals with advanced non Hodgkin's lymphoma clinical symptoms by using our model under the modified Bagdonavičius-Nikulin goodness-of-fit test statistic. Based on the MLEs, the modified Bagdonavičius-Nikulin goodness-of-fit test recovers the loss in information while the grouping data follow chi-square distributions. The corresponding elements of the modified Bagdonavičius-Nikulin goodness-of-fit criteria tests are explicitly derived.

A random variable (RV) $Z$ is said to have the log-logistic (LL) distribution if its survival function (SF) is written as

$$
\bar{S}_{b}(z)=1-G_{b}(z)=\frac{1}{1+z^{b}},
$$

where $z \geq 0$ and $b>0$ is a shape parameter, and $G_{b}(z)$ refers to the cumulative distribution functions (CDF) of the LL model. The corresponding probability density function (PDF) of (1.1) is given by

$$
g_{b}(z)=\frac{b z^{b-1}}{\left(1+z^{b}\right)^{2}} .
$$

The PDF in (1.2) is a special case from the well-know Burr type XII (BXII) model (Burr 1942, 1968, 1973; Rodriguez, 1977; Tadikamalla, 1980). Based on the family of Yousof et al. (2018) and using (1.1), the CDF of the Exponential Generalized log-logistic (EG-LL) is then defined by,

$$
F_{\beta, b, a}(z)=1-\exp \left\{-a\left[\left(1+z^{b}\right)^{\beta}-1\right]\right\}
$$

where $z \geq 0$ and $\beta>0, b>0, a>0$. The corresponding PDF is given by,

$$
f_{\beta, b, a}(z)=\beta a b z^{b-1}\left(1+z^{b}\right)^{\beta-1} \exp \left\{-a\left[\left(1+z^{b}\right)^{\beta}-1\right]\right\} .
$$

For $a=1$, the EG-LL model reduces to the two-parameter EG-LL model. For $\beta=1$, the EGLL model reduces to the two-parameter exponential $\log$-logistic (E-LL) model. For $\beta=a=1$, the 
EG-LL model reduces to the one-parameter E-LL model. Figure 1 below gives some plots for the PDF and hazard rate function (HRF) for the EG-LL model. From Figure 1(a) shows that the new PDF can have many useful shapes. From Figure 1(b) we conclude that the new HRF can be "bathtub $(\beta=5, b=0.01, a=0.55)$ ", "constant $(\beta=1, b=0.01, a=1)$ ", "J-HRF $(\beta=2, b=2, a=3.5)$ ", "increasing $(\beta=5, b=0.01, a=0.95)$ ", "decreasing $(\beta=3, b=0.01, a=0.25)$ " and "decreasingconstant $(\beta=3, b=0.01, a=0.55)$ ".

The $\mathrm{p}^{\text {th }}$ ordinary moment of $Z$ where $Z \sim \operatorname{EG}-\operatorname{LL}(\beta, b, a)$ is given by,

$$
\mu_{p}^{\prime}=\mathbf{E}\left(Z^{p}\right)=\int_{-\infty}^{\infty} z^{p} f(z) d z
$$

Then, using (1.5) we obtain

$$
\mu_{p}^{\prime}=\sum_{\kappa_{4}=0}^{\infty} u_{\kappa_{4}} \kappa_{4}^{*} B\left(1+\frac{p}{b}, \kappa_{4}^{*}-\frac{p}{b}\right)
$$

where $\kappa_{4}^{*}=1+\kappa_{4}, p<\kappa_{4}^{*} b, B\left(a_{1}, a_{2}\right)=\int_{0}^{\infty} t^{a_{1}-1}(1+t)^{-\left(a_{1}+a_{2}\right)} d t$ and

$$
u_{\kappa_{4}}=\sum_{\kappa_{1}, \kappa_{2}, \kappa_{3}=0}^{\infty} \frac{(-1)^{1+\kappa_{1}+\kappa_{2}+\kappa_{3}+\kappa_{4}}}{\Gamma\left(1+\kappa_{1}\right) \Gamma \kappa_{4}^{*}}\left(\begin{array}{l}
\kappa_{1} \\
\kappa_{2}
\end{array}\right)\left(\begin{array}{c}
\beta\left(\kappa_{2}-\kappa_{1}\right) \\
\kappa_{3}
\end{array}\right)\left(\begin{array}{c}
1+\kappa_{3} \\
\kappa_{4}
\end{array}\right)
$$

is the beta function of the second type. By setting $p=1$ in (1.5), we get the mean of $Z$. The $p^{t h}$ incomplete moment $\left(\varsigma_{p}(t)\right)$ of $Z$ can be expressed as,

$$
\varsigma_{p}(t)=\int_{-\infty}^{t} z^{p} f(z) d z=\sum_{\kappa_{4}=0}^{\infty} u_{\kappa_{4}} \kappa_{4}^{*} B\left(t^{b} ; 1+\frac{p}{b}, \kappa_{4}^{*}-\frac{p}{b}\right),
$$

where $p<\kappa_{4}^{*} b$ and $B\left(q ; a_{1}, a_{2}\right)=\int_{0}^{q} t^{a_{1}-1}(1+t)^{-\left(a_{1}+a_{2}\right)} d t$ is the incomplete beta function of the second type. The moment generating function $M_{Z}(t)=\mathbf{E}(\exp (t Z))$ of $Z$ can be easily derived. Then, the $p^{t h}$ moment of the reversed residual life of $Z$ becomes

$$
A_{p}(t)=F^{-1}(t) \sum_{r=0}^{\infty} l_{\kappa_{4}} \kappa_{4}^{*} B\left(t^{b} ; \kappa_{4}^{*}-\frac{p}{b}, 1+\frac{p}{b}\right),
$$

where

$$
l_{\kappa_{4}}=u_{\kappa_{4}} \sum_{r=0}^{p}(-1)^{r}\left(\begin{array}{l}
p \\
r
\end{array}\right) t^{p-r} .
$$

The proposed EG-LL model has only three parameters. However, in the applications to real data sets, all competitive distributions have at least three parameters (see comparing models under complete samples in Section 4). The EG-LL model with this advantage of a smaller number of parameters is the favorable statistical modeling, especially if it gives a better fit under some goodness-of-fit statistics tests (Tables 5, 6 and 7). So, it is recommended to use the EG-LL model instead of all other 
competitive models. In statistical modeling and applied fields, the EG-LL model could be useful in the following cases,

- Modeling the asymmetric right skewed real-life data sets especially the right skewed and heavy tail real-life data sets.

- Modeling the symmetric and asymmetric right skewed real-life data sets especially in case of modeling a certain real-life data for the first time.

- In reliability analysis, some physics studies and many engineering applications, the EG-LL model can be applied in modeling the breaking stress reliability data. As shown in Tables 5 and 6 , the EG-LL model shows its superiority against the log-logistic, the exponential log-logistic, the Burr type XII, the Weibull log-logistic, the Marshall-Olkin Burr type XII, the Topp Leone Burr type XII, the Zografos-Balakrishnan Burr type XII, Five Parameters beta Burr type XII, Beta Burr Burr type XII, and the Beta exponentiated Burr type XII, the Five Parameters Kumaraswamy Burr type XII and Kumaraswamy Burr type XII distributions.

- In survival analysis of guinea pigs, the EG-LL model can be chosen in modeling the survival times data. Based on Table 8, the EG-LL model performs better than the log-logistic, the exponential log-logistic, the Burr type XII, the Weibull log-logistic, the Marshall-Olkin Burr type XII, the Topp Leone Burr type XII, the Zografos-Balakrishnan Burr type XII, the Five Parameters beta Burr type XII, the Beta Burr Burr type XII, the Beta exponentiated Burr type XII, the Five Parameters Kumaraswamy Burr type XII and the Kumaraswamy Burr type XII distributions.

- In the medicine, the EG-LL model can be applied for modeling the acute myelogenous leukemia data. The new model showed its superiority against many competitive models such as the loglogistic, the exponential log-logistic, the Burr type XII, the Weibull log-logistic, the MarshallOlkin Burr type XII, the Topp Leone Burr type XII, the Zografos-Balakrishnan Burr type XII, the Five Parameters beta Burr type XII, the Beta Burr Burr type XII, the Beta exponentiated Burr type XII, the Five Parameters Kumaraswamy Burr type XII and the Kumaraswamy Burr type XII distributions as shown in Table 9 and Table 10.

\section{Non-Bayesian estimation methods under complete samples schemes}

\subsection{The MLE method}

Suppose that $z_{1}, z_{2}, \ldots, z_{n}$ is a RS from the EG-LL model with parameter vector $\underline{\boldsymbol{\Phi}}=(\beta, a, b)^{\top}$. Then the log-likelihood function $\left(\mathcal{L}_{n}(\underline{\Phi})\right)$ for $\underline{\boldsymbol{\Phi}}$ is given by,

$$
\mathcal{L}_{n}(\underline{\boldsymbol{\Phi}})=n \log \beta+n \log a+n \log b+(\beta-1) \sum_{\zeta=1}^{n} \log \left(1+z_{[s: n]}^{b}\right)-a \sum_{\zeta=1}^{n}\left[\left(1+z_{[s: n]}^{b}\right)^{\beta}-1\right],
$$

where the above $\mathcal{L}_{n}(\underline{\Phi})$ can be maximized numerically via SAS (PROC NLMIXED) or R (optim) or Ox program (via sub-routine MaxBFGS), among others. The components of the score vector

$$
\mathbf{U}(\underline{\Phi})=\frac{\partial \mathcal{L}}{\partial \underline{\boldsymbol{\Phi}}}=\left(\frac{\partial \mathcal{L}_{n}(\underline{\boldsymbol{\Phi}})}{\partial \beta}, \frac{\partial \mathcal{L}_{n}(\underline{\boldsymbol{\Phi}})}{\partial a}, \frac{\partial \mathcal{L}_{n}(\underline{\boldsymbol{\Phi}})}{\partial b}\right)^{\top},
$$

can easily be derived. 


\subsection{CVME method}

The CVME of the parameters $\beta, b$ and $a$ are obtained via minimizing the following expression with respect to the parameters $\beta, b$ and $a$ respectively, where

$$
\operatorname{CVME}_{(\underline{\boldsymbol{\Phi}})}=\frac{1}{12} n^{-1}+\sum_{\zeta=1}^{n}\left[F_{\underline{\Phi}}\left(z_{[\zeta: n]}\right)-c_{(\varsigma, n)}\right]^{2},
$$

and where $c_{(\varsigma, n)}=(2 \varsigma-1) / 2 n$ and

$$
\operatorname{CVME}_{(\underline{\Phi})}=\frac{1}{12} n^{-1}+\sum_{\varsigma=1}^{n}\left[\left(1-\exp \left\{-a\left[\left(1+z_{[S: n]}^{b}\right)^{\beta}-1\right]\right\}\right)-c_{(\varsigma, n)}\right]^{2} .
$$

The CVME of the parameters $\beta, b$ and $a$ are obtained by solving the two following non-linear equations as

$$
\begin{aligned}
& \sum_{S=1}^{n}\left[\left(1-\exp \left\{-a\left[\left(1+z_{[S: n]}^{b}\right)^{\beta}-1\right]\right\}\right)-c_{(\zeta, n)}\right] \nabla_{(\beta)}\left(z_{[S: n]} ; \underline{\Phi}\right)=0, \\
& \sum_{S=1}^{n}\left[\left(1-\exp \left\{-a\left[\left(1+z_{[S: n]}^{b}\right)^{\beta}-1\right]\right\}\right)-c_{(\zeta, n)}\right] \nabla_{(b)}\left(z_{[S: n]} ; \underline{\Phi}\right)=0, \\
& \sum_{S=1}^{n}\left[\left(1-\exp \left\{-a\left[\left(1+z_{[S: n]}^{b}\right)^{\beta}-1\right]\right\}\right)-c_{(S, n)}\right] \nabla_{(a)}\left(z_{[S: n]} ; \underline{\Phi}\right)=0,
\end{aligned}
$$

where

$$
\begin{aligned}
& \nabla_{(\beta)}\left(z_{[S: n]} ; \underline{\boldsymbol{\Phi}}\right)=\frac{\partial F_{\underline{\Phi}}\left(z_{[S: n]}\right)}{\partial \beta}, \\
& \nabla_{(b)}\left(z_{[S: n]} ; \underline{\mathbf{\Phi}}\right)=\frac{\partial F_{\underline{\Phi}}\left(z_{[S: n]}\right)}{\partial b}, \\
& \nabla_{(a)}\left(z_{[S: n]} ; \underline{\mathbf{\Phi}}\right)=\frac{\partial F_{\underline{\Phi}}\left(z_{[S: n]}\right)}{\partial a} .
\end{aligned}
$$

\subsection{OLSE method}

Let $F_{\Phi}\left(z_{[s: n]}\right)$ denote the CDF of the EG-LL model and let $z_{1: n}<z_{2: n}<\cdots<z_{n: n}$ be the $n$ ordered RS. The OLSEs are obtained upon minimizing,

$$
\operatorname{OLSE}(\underline{\Phi})=\sum_{S=1}^{n}\left[F_{\underline{\Phi}}\left(z_{[s: n]}\right)-v_{(\zeta, n)}\right]^{2} .
$$

Then, we have

$$
\operatorname{OLSE}(\underline{\boldsymbol{\Phi}})=\sum_{\zeta=1}^{n}\left[\left(1-\exp \left\{-a\left[\left(1+z_{[s: n]}^{b}\right)^{\beta}-1\right]\right\}\right)-v_{(\zeta, n)}\right]^{2}
$$


where $v_{(\varsigma, n)}=\varsigma / n+1$. The LSEs are obtained via solving the following non-linear equations,

$$
\begin{aligned}
& 0=\sum_{\zeta=1}^{n}\left[\left(1-\exp \left\{-a\left[\left(1+z_{[\varsigma: n]}^{b}\right)^{\beta}-1\right]\right\}\right)-v_{(\varsigma, n)}\right] \nabla_{(\beta)}\left(z_{[\zeta: n]} ; \underline{\mathbf{\Phi}}\right), \\
& 0=\sum_{S=1}^{n}\left[\left(1-\exp \left\{-a\left[\left(1+z_{[S: n]}^{b}\right)^{\beta}-1\right]\right\}\right)-v_{(\varsigma, n)}\right] \nabla_{(b)}\left(z_{[S: n]} ; \underline{\Phi}\right), \\
& 0=\sum_{S=1}^{n}\left[\left(1-\exp \left\{-a\left[\left(1+z_{[S: n]}^{b}\right)^{\beta}-1\right]\right\}\right)-v_{(\zeta, n)}\right] \nabla_{(a)}\left(z_{[S: n]} ; \underline{\mathbf{\Phi}}\right),
\end{aligned}
$$

where $\nabla_{(\beta)}\left(z_{[s: n]} ; \underline{\Phi}\right), \nabla_{(b)}\left(z_{[s: n]} ; \underline{\boldsymbol{\Phi}}\right)$ and $\nabla_{(a)}\left(z_{[\zeta: n]} ; \underline{\boldsymbol{\Phi}}\right)$ are defined above.

\subsection{WLSE method}

The WLSE is obtained by minimizing the function $\operatorname{WLSE}(\underline{\Phi})$ with respect to $\beta, b$ and $a$

$$
\operatorname{WLSE}(\underline{\boldsymbol{\Phi}})=\sum_{\zeta=1}^{n} \vartheta_{(\varsigma, n)}\left[F_{\underline{\Phi}}\left(z_{[\varsigma: n]}\right)-v_{(\varsigma, n)}\right]^{2}
$$

where

$$
\vartheta_{(\varsigma, n)}=\frac{\left[(1+n)^{2}(2+n)\right]}{[\varsigma(1+n-\varsigma)]} .
$$

The WLSEs are obtained by solving,

$$
\begin{aligned}
& 0=\sum_{\zeta=1}^{n}\left\{\left[\left(1-\exp \left\{-a\left[\left(1+z_{[S: n]}^{b}\right)^{\beta}-1\right]\right\}\right)-v_{(\varsigma, n)}\right] \vartheta_{(\varsigma, n)} \nabla_{(\beta)}\left(z_{[S: n]} ; \underline{\mathbf{\Phi}}\right)\right\}, \\
& 0=\sum_{\varsigma=1}^{n}\left\{\left[\left(1-\exp \left\{-a\left[\left(1+z_{[\varsigma: n]}^{b}\right)^{\beta}-1\right]\right\}\right)-v_{(\varsigma, n)}\right] \vartheta_{(\varsigma, n)} \nabla_{(b)}\left(z_{[\varsigma: n]} ; \underline{\mathbf{\Phi}}\right)\right\}, \\
& 0=\sum_{\varsigma=1}^{n}\left\{\left[\left(1-\exp \left\{-a\left[\left(1+z_{[s: n]}^{b}\right)^{\beta}-1\right]\right\}\right)-v_{(\varsigma, n)}\right] \vartheta_{(\varsigma, n)} \nabla_{(a)}\left(z_{[S: n]} ; \underline{\mathbf{\Phi}}\right)\right\} \text {. }
\end{aligned}
$$

\subsection{The ADE method}

The $\mathrm{ADE} \hat{\beta}_{(\mathrm{ADE})}, \hat{b}_{(\mathrm{ADE})}, \hat{a}_{(\mathrm{ADE})}$ are obtained by minimizing the function,

$$
\operatorname{ADE}_{\left(z_{[\zeta ; n], ;[-\zeta+1+n: n]}\right)}(\underline{\Phi})=-n-n^{-1} \sum_{\zeta=1}^{n}\left[\times\left\{\begin{array}{c}
(2 \varsigma-1) \\
\log F_{(\underline{\Phi})}\left(z_{[\zeta: n]}\right) \\
+\log \left[1-F_{(\underline{\Phi})}\left(z_{[-\varsigma+1+n: n]}\right)\right]
\end{array}\right\}\right] .
$$


The parameter estimates $\hat{\beta}_{(\mathrm{ADE})}, \hat{b}_{(\mathrm{ADE})}, \hat{a}_{(\mathrm{ADE})}$ are derived by solving the nonlinear equations,

$$
\begin{aligned}
& 0=\frac{\partial\left[\operatorname{ADE}_{\left(z_{[s: n]}, z_{[-\zeta+1+n: n]}\right)}(\underline{\Phi})\right]}{\partial \beta},
\end{aligned}
$$

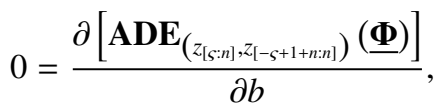

$$
\begin{aligned}
& 0=\frac{\partial\left[\mathbf{A D E}_{\left(z_{[;: n]}, z_{[-S+1+n: n]}\right)}(\underline{\boldsymbol{\Phi}})\right]}{\partial a} .
\end{aligned}
$$

\subsection{The ADE (R-T) method}

The $\mathrm{ADE}(\mathrm{R}-\mathrm{T}) \hat{\beta}_{\left(\mathrm{ADE}_{(\mathrm{R}-\mathrm{T})}\right)}, \hat{b}_{\left(\mathrm{ADE}_{(\mathrm{R}-\mathrm{T})}\right)}, \hat{a}_{\left(\mathrm{ADE}_{(\mathrm{R}-\mathrm{T})}\right)}$ are obtained by minimizing,

$$
\begin{aligned}
\operatorname{ADE}_{(\mathrm{R}-\mathrm{T})}\left(z_{\left[[: n: n], z_{[-\zeta+1+n: n]}\right)}(\underline{\boldsymbol{\Phi}})\right. & =\frac{1}{2} n-2 \sum_{\zeta=1}^{n} F_{(\underline{\boldsymbol{\Phi}})}\left(z_{[\zeta: n]}\right) \\
& -\frac{1}{n} \sum_{\zeta=1}^{n}(2 \varsigma-1)\left\{\log \left[1-F_{(\underline{\Phi})}\left(z_{[-\zeta+1+n: n]}\right)\right]\right\} .
\end{aligned}
$$

The estimates $\hat{\beta}_{\left(\mathrm{ADE}_{(\mathrm{R}-\mathrm{T})}\right)}, \hat{b}_{\left(\mathrm{ADE}_{(\mathrm{R}-\mathrm{T})}\right)}, \hat{a}_{\left(\mathrm{ADE}_{(\mathrm{R}-\mathrm{T})}\right)}$ follow by solving the nonlinear equations,

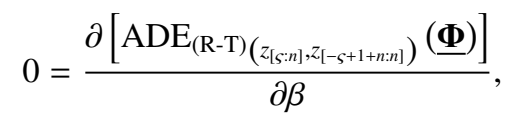

$$
\begin{aligned}
& 0=\frac{\partial\left[\operatorname{ADE}_{(\mathrm{R}-\mathrm{T})}\left(z_{\left[[: n], z_{[-\zeta+1+n: n]}\right)}(\underline{\boldsymbol{\Phi}})\right]\right.}{\partial b}, \\
& 0=\frac{\partial\left[\operatorname{ADE}_{(\mathrm{R}-\mathrm{T})}\left(z_{\left.[;: n], z_{[-\zeta+1+n: n]}\right)}(\underline{\boldsymbol{\Phi}})\right]\right.}{\partial a} .
\end{aligned}
$$

\subsection{The $A D E(L-T)$ method}

The $\mathrm{ADE}(\mathrm{L}-\mathrm{T}) \hat{\beta}_{\left(\mathrm{ADE}_{(\mathrm{L}-\mathrm{T})}\right)}, \hat{b}_{\left(\mathrm{ADE}_{(\mathrm{L}-\mathrm{T})}\right)}, \hat{a}_{\left(\mathrm{ADE}_{(\mathrm{L}-\mathrm{T})}\right)}$ are obtained by minimizing,

$$
\operatorname{ADE}_{(\mathrm{L}-\mathrm{T})\left(z_{[;: n]}\right)}(\underline{\boldsymbol{\Phi}})=-\frac{3}{2} n+2 \sum_{\zeta=1}^{n} F_{(\underline{\Phi})}\left(z_{[\zeta: n]}\right)-\frac{1}{n} \sum_{\zeta=1}^{n}(2 \varsigma-1) \log F_{(\underline{\Phi})}\left(z_{[\zeta: n]}\right) .
$$

The estimates $\hat{\beta}_{\left(\operatorname{ADE}_{(L-T)}\right)}, \hat{b}_{\left(\operatorname{ADE}_{(L-T)}\right)}, \hat{a}_{\left(\operatorname{ADE}_{(L-T)}\right)}$ follow by solving the nonlinear equations,

$$
\begin{aligned}
& 0=\frac{\partial\left[\operatorname{ADE}_{(\mathrm{L}-\mathrm{T})}\left(z_{[;: n])}(\underline{\Phi})\right]\right.}{\partial \beta}, \\
& 0=\frac{\partial\left[\operatorname{ADE}_{(\mathrm{L}-\mathrm{T})}{\left(z_{[[;: n]}\right)}_{\partial b}(\underline{\boldsymbol{\Phi}})\right]}{\partial}, \\
& 0=\frac{\partial\left[\operatorname{ADE}_{(\mathrm{L}-\mathrm{T})}\left(\mathcal{z}_{[S: n])}(\underline{\boldsymbol{\Phi}})\right]\right.}{\partial a} \text {. }
\end{aligned}
$$




\section{Comparing the non-Bayesian estimation methods under complete samples schemes via a simulation study}

A numerical simulation is performed to compare the classical estimation methods. The simulation study is based on $\mathrm{N}=1000$ generated data sets from the EG-LL version where $n=50,100,300$ and 500. The simulation is performed in terms of bias $\left(\operatorname{BIAS}_{(\underline{\Phi})}\right)$, the root mean-standard error $\left(\operatorname{RMSE}_{(\underline{\Phi})}\right)$, the mean of the absolute difference between the theoretical and the estimates $\left(D_{\mathrm{abs}}\right)$, and the maximum absolute difference between the true parameters and estimates $\left(D_{\max }\right)$, where

$$
\begin{aligned}
& \operatorname{BIAS}_{(\beta)}=\frac{1}{B} \sum_{\zeta=1}^{B}(\hat{\beta}-\beta) \\
& \operatorname{BIAS}_{(b)}=\frac{1}{B} \sum_{\zeta=1}^{B}(\hat{b}-b) \\
& \operatorname{BIAS}_{(a)}=\frac{1}{B} \sum_{\zeta=1}^{B}(\hat{a}-a) \\
& \operatorname{RMSE}_{(\beta)}=\sqrt{\frac{1}{B} \sum_{\zeta=1}^{B}(\hat{\beta}-\beta)^{2}}, \\
& \operatorname{RMSE}_{(a)}=\sqrt{\frac{1}{B} \sum_{\zeta=1}^{B}(\hat{a}-a)^{2}}, \\
& \operatorname{RMSE}_{(b)}=\sqrt{\frac{1}{B} \sum_{\zeta=1}^{B}(\hat{b}-b)^{2}}, \\
& D_{\mathrm{abs}}=\frac{1}{n B} \sum_{\varsigma=1}^{B} \sum_{j=1}^{n}\left|F_{\underline{\Phi}}\left(z_{\varsigma j}\right)-F_{(\hat{\beta}, \hat{b}, \hat{a})}\left(\tau_{\varsigma j}\right)\right| \text {, } \\
& D_{\max }=\frac{1}{B} \sum_{\zeta=1}^{B} \max _{j}\left|F_{\underline{\Phi}}\left(z_{\varsigma j}\right)-F_{(\hat{\beta}, \hat{b}, \hat{a})}\left(z_{\varsigma j}\right)\right| .
\end{aligned}
$$

From Tables $1-3$ we note that,

- The $\operatorname{BIAS}_{(\underline{\Phi})}$ tend to 0 when $n$ increases and tent to $\infty$ which means that all estimators are nonbiased.

- The $\operatorname{RMSE}_{(\underline{\Phi})}$ tend to 0 , when $n$ increases and tent to $\infty$ which means incidence of consistency property.

- The MLE method is the best among all the non-Bayesian estimations methods.

- The $\operatorname{BIAS}_{(\Phi)}$ takes positive and sometimes negative values.

The following results are based on Table 1,

- For $n=50 \mid \beta=0.9, b=0.9, a=0.9$, the MLE method is the best with RMSE=0.07860,0.09449, 0.13275 . 
- For $n=100 \mid \beta=0.9, b=0.9, a=0.9$, the MLE method is the best with RMSE $=0.05537,0.06685$, 0.09235 .

- For $n=300 \mid \beta=0.9, b=0.9, a=0.9$, the MLE method is the best with RMSE=0.03037, 0.03696, 0.05179 .

- For $n=500 \mid \beta=0.9, b=0.9, a=0.9$, the MLE method is the best with RMSE=0.02306, 0.02795, 0.03919 .

Based on Tables 2, the following results are,

- For $n=50 \mid \beta=1.2, b=1.5, a=0.1$, the MLE method is the best with RMSE $=0.05641,0.07782$, 0.01477 .

- For $n=100 \mid \beta=1.2, b=1.5, a=0.1$, the MLE method is the best with RMSE=0.04007, 0.05464, 0.01062 .

- For $n=300 \mid \beta=1.2, b=1.5, a=0.1$, the MLE method is the best with RMSE $=0.02135,0.02924$, 0.00562 .

- For $n=500 \mid \beta=1.2, b=1.5, a=0.1$, the MLE method is the best with RMSE=0.01690, $0.02293,0.00446$.

Based on Tables 3, the following results are,

- For $n=50 \mid \beta=0.2, b=0.6, a=1.2$ the MLE method is the best with RMSE $=0.00959,0.05527$, 0.17868 .

- For $n=100 \mid \beta=0.2, b=0.6, a=1.2$, the MLE method is the best with RMSE=0.00634, 0.03677, 0.12063 .

- For $n=300 \mid \beta=0.2, b=0.6, a=1.2$, the MLE method is the best with RMSE=0.00373, 0.02192, 0.06960 .

- For $n=500 \mid \beta=0.2, b=0.6, a=1.2$, the MLE method is the best with RMSE=0.00284, 0.01649, 0.05288 .

Based on the results of Tables 1-3, the MLE method is considered for modeling the real-life data sets in the following Section.

\section{Comparing models under complete samples}

For comparing models under complete samples case, we provide three real data applications. These applications, also illustrate the importance, potentiality and flexibility of the EG-LL model in modeling real data. For these three data applications, we compare the EG-LL distribution with the Burr type XII (BXII), the Marshall-Olkin Burr XII (MOBXII), the Topp Leone Burr XII (TLBXII), the Zografos-Balakrishnan Burr XII (ZBBXII), Five Parameters beta Burr XII (FBBXII), Beta Burr XII (BBXII), Beta exponentiated Burr XII (BEBXII), Five Parameters Kumaraswamy Burr XII (FKwBXII), Kumaraswamy Burr XII (KwBXII), exponential log-logistic (E-LL). and Weibull log-logistic (WLL) distributions given in Yousof et al. (2018), Altun et al. (2018 a, b) and Yousof et al. (2019). 
Table 1: Simulation results for parameters $\beta=0.9, b=0.9$ and $a=0.9$

\begin{tabular}{|c|c|c|c|c|c|c|c|c|c|}
\hline & \multicolumn{4}{|c|}{ BIAS } & \multicolumn{3}{|c|}{ RMSE } & \multicolumn{2}{|c|}{$D$} \\
\hline & $\mathrm{n}$ & $\hat{\beta}$ & $\hat{b}$ & $\hat{a}$ & $\hat{\beta}$ & $\hat{b}$ & $\hat{a}$ & $D_{\text {abs }}$ & $D_{\max }$ \\
\hline MLE & 50 & 0.00880 & 0.01193 & 0.01097 & 0.07860 & 0.09449 & 0.13275 & 0.00642 & 0.01195 \\
\hline CVM & & 0.00577 & -0.01521 & 0.00963 & 0.10507 & 0.12259 & 0.15231 & 0.00503 & 0.00802 \\
\hline OLS & & -0.00971 & 0.00007 & -0.01301 & 0.09766 & 0.12525 & 0.14005 & 0.00744 & 0.01109 \\
\hline WLS & & -0.00910 & 0.07468 & -0.01814 & 0.08258 & 0.14968 & 0.13148 & 0.01323 & 0.02385 \\
\hline $\mathrm{ADE}$ & & 0.00697 & -0.01483 & 0.01361 & 0.08885 & 0.10349 & 0.13690 & 0.00661 & 0.01117 \\
\hline $\mathrm{ADE}(\mathrm{R}-\mathrm{T})$ & & 0.00581 & 0.00622 & 0.01150 & 0.08320 & 0.12107 & 0.13258 & 0.00537 & 0.01060 \\
\hline $\mathrm{ADE}(\mathrm{L}-\mathrm{T})$ & & 0.01167 & 0.00197 & 0.01786 & 0.12321 & 0.16005 & 0.17147 & 0.00936 & 0.01593 \\
\hline MLE & 100 & 0.00699 & 0.01029 & 0.00898 & 0.05537 & 0.06685 & 0.09235 & 0.00521 & 0.00982 \\
\hline CVM & & 0.00442 & -0.00288 & 0.00663 & 0.07274 & 0.08295 & 0.10538 & 0.00357 & 0.00501 \\
\hline OLS & & -0.00717 & -0.00225 & -0.00996 & 0.06986 & 0.08312 & 0.10041 & 0.00557 & 0.00871 \\
\hline WLS & & -0.00589 & 0.04671 & -0.01328 & 0.05798 & 0.08998 & 0.09585 & 0.00862 & 0.01566 \\
\hline $\mathrm{ADE}$ & & 0.00140 & -0.00832 & 0.00356 & 0.06291 & 0.07201 & 0.09619 & 0.00182 & 0.00452 \\
\hline ADE(R-T) & & 0.00105 & 0.00171 & 0.00278 & 0.05917 & 0.08698 & 0.09372 & 0.00117 & 0.00327 \\
\hline $\mathrm{ADE}(\mathrm{L}-\mathrm{T})$ & & 0.00606 & 0.00430 & 0.00870 & 0.08373 & 0.10625 & 0.11633 & 0.00471 & 0.00919 \\
\hline MLE & 300 & 0.00188 & 0.00189 & 0.00290 & 0.03037 & 0.03696 & 0.05179 & 0.00152 & 0.00264 \\
\hline CVM & & 0.00235 & -0.00202 & 0.00360 & 0.04187 & 0.04856 & 0.06095 & 0.00192 & 0.00269 \\
\hline OLS & & -0.00170 & -0.00296 & -0.00209 & 0.04081 & 0.04869 & 0.05939 & 0.00128 & 0.00249 \\
\hline WLS & & -0.00148 & 0.02383 & -0.00488 & 0.03248 & 0.04915 & 0.05480 & 0.00416 & 0.00724 \\
\hline $\mathrm{ADE}$ & & 0.00057 & -0.00512 & 0.00179 & 0.03611 & 0.04102 & 0.05595 & 0.00101 & 0.00288 \\
\hline RTADE & & -0.00005 & -0.00224 & 0.00068 & 0.03297 & 0.04572 & 0.05275 & 0.00040 & 0.00175 \\
\hline LEADE & & 0.00328 & 0.00036 & 0.00475 & 0.04861 & 0.06292 & 0.06783 & 0.00258 & 0.00504 \\
\hline MLE & 500 & 0.00070 & 0.00237 & 0.00038 & 0.02306 & 0.02795 & 0.039190 & 0.00056 & 0.00115 \\
\hline CVM & & -0.00047 & 0.00011 & -0.00068 & 0.03107 & 0.03619 & 0.04501 & 0.00037 & 0.00054 \\
\hline OLS & & -0.00122 & -0.00053 & -0.00171 & 0.03283 & 0.03623 & 0.04760 & 0.00094 & 0.00151 \\
\hline WLS & & 0.00026 & 0.01510 & -0.00087 & 0.02519 & 0.03543 & 0.04236 & 0.00260 & 0.00408 \\
\hline ADE & & -0.00078 & -0.00027 & -0.00108 & 0.02783 & 0.03188 & 0.04259 & 0.00060 & 0.00199 \\
\hline $\mathrm{ADE}(\mathrm{R}-\mathrm{T})$ & & -0.00049 & 0.00037 & -0.00067 & 0.02594 & 0.03742 & 0.04109 & 0.00038 & 0.00158 \\
\hline $\mathrm{ADE}(\mathrm{L}-\mathrm{T})$ & & -0.00049 & 0.00278 & -0.00064 & 0.03543 & 0.04626 & 0.04928 & 0.00052 & 0.00202 \\
\hline
\end{tabular}

Data set I: called breaking stress data $\{0.98,5.56,5.08,0.39,1.57,3.19,4.90,2.93,2.85,2.77$, 2.76, 1.73, 2.48, 3.68, 1.08, 3.22, 3.75, 3.22, 3.70, 2.74, 2.73, 2.50, 3.60, 3.11, 3.27, 2.87, 1.47, 3.11, $4.42,2.40,3.15,2.67,3.31,2.81,2.56,2.17,4.91,1.59,1.18,2.48,2.03,1.69,2.43,3.39,3.56,2.83$, $3.68,2.00,3.51,0.85,1.61,3.28,2.95,2.81,3.15,1.92,1.84,1.22,2.17,1.61,2.12,3.09,2.97,4.20$, $2.35,1.41,1.59,1.12,1.69,2.79,1.89,1.87,3.39,3.33,2.55,3.68,3.19,1.71,1.25,4.70,2.88,2.96$, $2.55,2.59,2.97,1.57,2.17,4.38,2.03,2.82,2.53,3.31,2.38,1.36,0.81,1.17,1.84,1.80,2.05,3.65\}$. This data set consists of 100 observations of breaking stress of carbon fibres (in Gba) given by Nichols and Padgett (2006).

Data set II: called survival times in days of 72 guinea pigs infected with the virulent tubercle bacilli $\{0.1,0.33,0.44,0.56,0.59,0.72,0.74,0.77,0.92,0.93,0.96,1,1,1.02,1.05,1.07,07,1.08$, $1.08,1.08,1.09,1.12,1.13,1.15,1.16,1.2,1.21,1.22,1.22,1.24,1.3,1.34,1.36,1.39,1.44,1.46$, $1.53,1.59,1.6,1.63,1.63,1.68,1.71,1.72,1.76,1.83,1.95,1.96,1.97,2.02,2.13,2.15,2.16,2.22$, $2.3,2.31,2.4,2.45,2.51,2.53,2.54,2.54,2.78,2.93,3.27,3.42,3.47,3.61,4.02,4.32,4.58,5.55\}$, this data was originally observed and reported by Bjerkedal (1960).

Data set III $\{65,156,100,134,16,108,121,4,39,143,56,26,22,1,1,5,65,56,65,17,7,16$, $22,3,4,2,3,8,4,3,30,4,43\}$ called the leukaemia data. This real data set gives the survival times, in weeks, of 33 patients suffering from acute myelogenous leukemia. Those real data sets were recently analysed by Altun et al. (2018a), Altun et al. (2018b), Abouelmagd et al. (2019), Gad et al. (2019) and Elsayed and Yousof $(2019,2021)$. The total time test (TTT) plot (Aarset, 1987) is an important 
Table 2: Simulation results for parameters $\beta=1.2, b=1.5$ and $a=0.1$

\begin{tabular}{|c|c|c|c|c|c|c|c|c|c|}
\hline & & \multicolumn{3}{|c|}{ BIAS } & \multicolumn{3}{|c|}{ RMSE } & \multicolumn{2}{|c|}{$D$} \\
\hline & $\mathrm{n}$ & $\hat{\beta}$ & $\hat{b}$ & $\hat{a}$ & $\hat{\beta}$ & $\hat{b}$ & $\hat{a}$ & $D_{\text {abs }}$ & $D_{\max }$ \\
\hline MLE & 50 & 0.00834 & 0.01278 & 0.00193 & 0.05641 & 0.07782 & 0.01477 & 0.01299 & 0.02089 \\
\hline CVM & & 0.00530 & 0.01033 & 0.00159 & 0.07635 & 0.11647 & 0.01727 & 0.00984 & 0.01581 \\
\hline OLS & & -0.00803 & -0.01005 & -0.00126 & 0.07577 & 0.11459 & 0.01697 & 0.01042 & 0.01696 \\
\hline WLS & & -0.00743 & 0.00133 & -0.00244 & 0.06222 & 0.08810 & 0.01536 & 0.00966 & 0.01450 \\
\hline $\mathrm{ADE}$ & & 0.00027 & -0.00059 & 0.00085 & 0.06888 & 0.09799 & 0.01664 & 0.00209 & 0.00323 \\
\hline $\mathrm{ADE}(\mathrm{R}-\mathrm{T})$ & & -0.00023 & 0.00041 & 0.00057 & 0.06344 & 0.09141 & 0.01580 & 0.00143 & 0.00228 \\
\hline $\mathrm{ADE}(\mathrm{L}-\mathrm{T})$ & & 0.00779 & 0.00793 & 0.0214 & 0.09101 & 0.12964 & 0.01953 & 0.01174 & 0.01867 \\
\hline MLE & 100 & 0.00388 & 0.00623 & 0.00088 & 0.04007 & 0.05464 & 0.01062 & 0.00611 & 0.00987 \\
\hline CVM & & 0.00192 & 0.00403 & 0.00064 & 0.05382 & 0.08093 & 0.01214 & 0.00382 & 0.00614 \\
\hline OLS & & -0.00396 & -0.00469 & -0.00066 & 0.05367 & 0.08076 & 0.01196 & 0.00516 & 0.00833 \\
\hline WLS & & -0.00050 & 0.00761 & -0.00067 & 0.04276 & 0.05937 & 0.01098 & 0.00088 & 0.00175 \\
\hline $\mathrm{ADE}$ & & 0.00303 & 0.00369 & 0.00110 & 0.04623 & 0.06623 & 0.01106 & 0.00541 & 0.00863 \\
\hline $\mathrm{ADE}(\mathrm{R}-\mathrm{T})$ & & -0.00046 & -0.00053 & 0.00021 & 0.04165 & 0.05978 & 0.01037 & 0.00068 & 0.00118 \\
\hline $\operatorname{ADE}(\mathrm{L}-\mathrm{T})$ & & 0.00285 & 0.00290 & 0.00084 & 0.06320 & 0.090240 & 0.01354 & 0.00447 & 0.00719 \\
\hline MLE & 300 & 0.00184 & 0.00235 & 0.00052 & 0.02135 & 0.02924 & 0.00562 & 0.00298 & 0.00473 \\
\hline CVM & & 0.00204 & 0.00326 & 0.00053 & 0.02935 & 0.04389 & 0.00660 & 0.00337 & 0.00543 \\
\hline OLS & & -0.00262 & -0.00360 & -0.00050 & 0.02897 & 0.04344 & 0.00645 & 0.00372 & 0.00602 \\
\hline WLS & & -0.00224 & 0.00128 & -0.00085 & 0.02272 & 0.03136 & 0.00588 & 0.00290 & 0.00428 \\
\hline $\mathrm{ADE}$ & & 0.00026 & 0.00023 & 0.00018 & 0.02610 & 0.03708 & 0.00625 & 0.00066 & 0.00113 \\
\hline RTADE & & 0.00032 & 0.00049 & 0.00015 & 0.02488 & 0.03581 & 0.00612 & 0.00035 & 0.00064 \\
\hline LEADE & & 0.00283 & 0.00315 & 0.00071 & 0.03420 & 0.04943 & 0.00731 & 0.00420 & 0.00679 \\
\hline MLE & 500 & -0.00006 & 0.00005 & -0.000001 & 0.01690 & 0.02293 & 0.00446 & 0.00002 & 0.00003 \\
\hline CVM & & -0.00004 & 0.00012 & 0.00004 & 0.02322 & 0.03464 & 0.00520 & 0.00011 & 0.00017 \\
\hline OLS & & -0.00059 & -0.00092 & -0.00005 & 0.02302 & 0.0343 & 0.00516 & 0.00071 & 0.00119 \\
\hline WLS & & 0.00023 & 0.00337 & -0.00014 & 0.01837 & 0.02535 & 0.00477 & 0.00083 & 0.00162 \\
\hline ADE & & -0.00047 & -0.00086 & -0.00002 & 0.02001 & 0.02843 & 0.00477 & 0.00056 & 0.00107 \\
\hline $\mathrm{ADE}(\mathrm{R}-\mathrm{T})$ & & 0.00005 & 0.00019 & 0.00011 & 0.01898 & 0.02727 & 0.00468 & 0.00014 & 0.00039 \\
\hline $\mathrm{ADE}(\mathrm{L}-\mathrm{T})$ & & 0.00019 & -0.00012 & 0.00011 & 0.02696 & 0.03887 & 0.00574 & 0.00033 & 0.00060 \\
\hline
\end{tabular}

graphical approach to verify whether the data can be applied to a specific distribution or not. The TTT plots the three real data sets as presented in Figure 2. This plot indicates that the empirical HRFs of data sets I, II are increasing and are bathtub for data set III.

We consider the following goodness-of-fit statistics: the Akaike information criterion $\left(\mathrm{C}_{1}\right)$, the Bayesian information criterion $\left(\mathrm{C}_{2}\right)$, the consistent Akaike information criterion $\left(\mathrm{C}_{3}\right)$ and the HannanQuinn information criterion $\left(\mathrm{C}_{4}\right)$, where

$$
\begin{aligned}
& \mathrm{C}_{1}=-2 \mathcal{L}(\underline{\hat{\boldsymbol{\Phi}}})+2 m, \mathrm{C}_{2}=-2 \mathcal{L}(\underline{\hat{\boldsymbol{\Phi}}})+m \log (n), \\
& \mathrm{C}_{3}=-2 \mathcal{L}(\underline{\hat{\boldsymbol{\Phi}}})+2 n m /(n-m-1) \text { and } \mathrm{C}_{4}=-2 \mathcal{L}(\underline{\hat{\boldsymbol{\Phi}}})+2 m \log [\log (n)],
\end{aligned}
$$

and where $m$ is the number of parameters, $n$ is the sample size, and $-2 \mathcal{L}(\underline{\hat{\boldsymbol{\Phi}}})$ is the maximized $\log$ likelihood. Generally, the smaller these statistics are, the better the fit. Tables 5, 7 and 9 give the MLEs, standard errors (SEs) and confidence interval (CIs) with for the data set I, II and III, respectively. Tables 6, 8 and 10 give the statistics $\mathrm{C}_{1}, \mathrm{C}_{2}, \mathrm{C}_{3}$ and $\mathrm{C}_{4}$ values for the data set I, II and III. Based on the values in Tables 6, 8 and 10 and Figures 3-7 the EG-LL model provide the best fit as compared to other competitive models in the four applications with small values for $\mathrm{C}_{1}, \mathrm{C}_{2}, \mathrm{C}_{3}$ and $\mathrm{C}_{4}$. Figure 8 presents profile likelihood plots (the first row is for data set $\mathbf{I}$, the second row is for data set II and the third row is for data set III). 
Table 3: Simulation results for parameters $\beta=0.1, b=0.6$ and $a=1.2$

\begin{tabular}{|c|c|c|c|c|c|c|c|c|c|}
\hline & \multicolumn{4}{|c|}{ BIAS } & \multicolumn{3}{|c|}{ RMSE } & \multicolumn{2}{|c|}{$D$} \\
\hline & $\mathrm{n}$ & $\hat{\beta}$ & $\hat{b}$ & $\hat{a}$ & $\hat{\beta}$ & $\hat{b}$ & $\hat{a}$ & $D_{\text {abs }}$ & $D_{\max }$ \\
\hline MLE & 50 & 0.00095 & 0.00459 & 0.01347 & 0.00959 & 0.05527 & 0.17868 & 0.00821 & 0.01257 \\
\hline CVM & & 0.00082 & 0.00496 & 0.01449 & 0.01257 & 0.07630 & 0.20491 & 0.00819 & 0.01254 \\
\hline OLS & & -0.00178 & -0.01037 & -0.0276 & 0.01224 & 0.07409 & 0.19862 & 0.01716 & 0.02620 \\
\hline WLS & & -0.00175 & -0.00196 & -0.03531 & 0.00987 & 0.05883 & 0.17895 & 0.01428 & 0.02137 \\
\hline $\mathrm{ADE}$ & & 0.00040 & 0.00091 & 0.01231 & 0.01081 & 0.06415 & 0.18822 & 0.00434 & 0.00837 \\
\hline $\mathrm{ADE}(\mathrm{R}-\mathrm{T})$ & & 0.00033 & 0.00191 & 0.00992 & 0.01018 & 0.06138 & 0.18222 & 0.00412 & 0.00848 \\
\hline $\mathrm{ADE}(\mathrm{L}-\mathrm{T})$ & & 0.00142 & 0.00347 & 0.02336 & 0.01459 & 0.08306 & 0.22839 & 0.01118 & 0.01899 \\
\hline MLE & 100 & 0.00066 & 0.00331 & 0.01114 & 0.00634 & 0.03677 & 0.12063 & 0.00615 & 0.00940 \\
\hline CVM & & 0.00068 & 0.00391 & 0.01193 & 0.00857 & 0.05175 & 0.14044 & 0.00667 & 0.01021 \\
\hline OLS & & -0.00064 & -0.00366 & -0.00927 & 0.00855 & 0.05189 & 0.13901 & 0.00590 & 0.00907 \\
\hline WLS & & -0.00074 & 0.00239 & -0.01627 & 0.00702 & 0.04178 & 0.12902 & 0.00464 & 0.00676 \\
\hline $\mathrm{ADE}$ & & 0.00008 & -0.00012 & 0.00353 & 0.00752 & 0.04507 & 0.12900 & 0.00094 & 0.00315 \\
\hline $\mathrm{ADE}(\mathrm{R}-\mathrm{T})$ & & 0.00037 & 0.00217 & 0.00817 & 0.00709 & 0.04274 & 0.12695 & 0.00400 & 0.00783 \\
\hline $\mathrm{ADE}(\mathrm{L}-\mathrm{T})$ & & 0.00115 & 0.00390 & 0.01920 & 0.01012 & 0.05760 & 0.16014 & 0.00968 & 0.01648 \\
\hline MLE & 300 & 0.00023 & 0.00116 & 0.00385 & 0.00373 & 0.02192 & 0.06960 & 0.00216 & 0.00330 \\
\hline CVM & & 0.00026 & 0.00146 & 0.00472 & 0.00480 & 0.02909 & 0.07838 & 0.00256 & 0.00392 \\
\hline OLS & & -0.00014 & -0.00077 & -0.00192 & 0.00478 & 0.02890 & 0.07812 & 0.00124 & 0.00190 \\
\hline WLS & & -0.00005 & 0.00442 & -0.00309 & 0.00388 & 0.02271 & 0.07280 & 0.00149 & 0.00248 \\
\hline $\mathrm{ADE}$ & & -0.00002 & -0.00040 & 0.00049 & 0.00438 & 0.02616 & 0.07534 & 0.00018 & 0.00180 \\
\hline RTADE & & 0.00023 & 0.00137 & 0.00441 & 0.00419 & 0.02529 & 0.07420 & 0.00236 & 0.00506 \\
\hline LEADE & & 0.00048 & 0.00188 & 0.00811 & 0.00549 & 0.03225 & 0.08634 & 0.00420 & 0.00796 \\
\hline MLE & 500 & 0.00004 & 0.00003 & 0.00059 & 0.00284 & 0.01649 & 0.05288 & 0.00027 & 0.00041 \\
\hline CVM & & 0.00009 & 0.00052 & 0.00161 & 0.00364 & 0.02206 & 0.05951 & 0.00089 & 0.00135 \\
\hline OLS & & -0.00019 & -0.00113 & -0.00296 & 0.00387 & 0.02342 & 0.06323 & 0.00183 & 0.00280 \\
\hline WLS & & -0.00002 & 0.00330 & -0.00221 & 0.00295 & 0.01744 & 0.05596 & 0.00119 & 0.00197 \\
\hline $\mathrm{ADE}$ & & 0.00001 & -0.00007 & 0.00064 & 0.00332 & 0.01988 & 0.05722 & 0.00013 & 0.00158 \\
\hline $\mathrm{ADE}(\mathrm{R}-\mathrm{T})$ & & -0.00016 & -0.00098 & -0.00234 & 0.00308 & 0.01855 & 0.05442 & 0.00151 & 0.00367 \\
\hline $\operatorname{ADE}(\mathrm{L}-\mathrm{T})$ & & 0.00016 & 0.00038 & 0.00265 & 0.00419 & 0.02445 & 0.06600 & 0.00126 & 0.00332 \\
\hline
\end{tabular}

\section{Censored MLE}

Suppose that $Z_{1}, Z_{2}, \ldots, Z_{n}$ is a random sample with right censoring from EG-LL( $\left.\underline{\boldsymbol{\Phi}}\right)$ distribution where

$$
z_{\varsigma}=\left.\min \left(Z_{\varsigma}, C_{\zeta}\right)\right|_{(\varsigma=1,2, \ldots, n)},
$$

is the minimum of the survival time $Z_{\zeta}$ and the censoring time $C_{\varsigma}$ for each subject in the sample. So, $z_{\varsigma}$ can be written in the form $\left(z_{\varsigma}, \nabla_{\varsigma}\right)_{\varsigma=1, \ldots, n}$, where $\nabla_{\varsigma}=1$ if $Z_{\zeta}$ is the moment of failure (complete observation) and $\nabla_{\varsigma}=0$ if $Z_{\varsigma}$ is the moment of censoring. The right censoring is assumed to be non-informative, so the expression of the likelihood function is

$$
(z, \underline{\mathbf{\Phi}})=\left.\prod_{\varsigma=1}^{n}\left[h_{\underline{\Phi}}\left(z_{\zeta}\right)\right]^{\nabla_{\zeta}} S_{\underline{\Phi}}\left(z_{\zeta}\right)\right|_{\left(\nabla_{\zeta}=1_{z_{\zeta}<C_{\zeta}}\right)},
$$

where $h_{\Phi}\left(z_{\zeta}\right)$ refers to the HRF and $S_{\Phi}\left(z_{\zeta}\right)$ refers to the survival function (SF). The log-likelihood function of $\operatorname{EG-LL}(\underline{\Phi})$ distribution is

$$
\mathcal{L}_{n}(\underline{\boldsymbol{\Phi}})=\sum_{\zeta=1}^{n} \nabla_{\varsigma}\left[\ln (\beta a b)+(b-1) \ln z_{\varsigma}+(\beta-1) \ln \left(1+z_{\varsigma}^{b}\right)\right]-a \sum_{\varsigma=1}^{n}\left[\left(1+z_{\varsigma}^{b}\right)^{\beta}-1\right],
$$



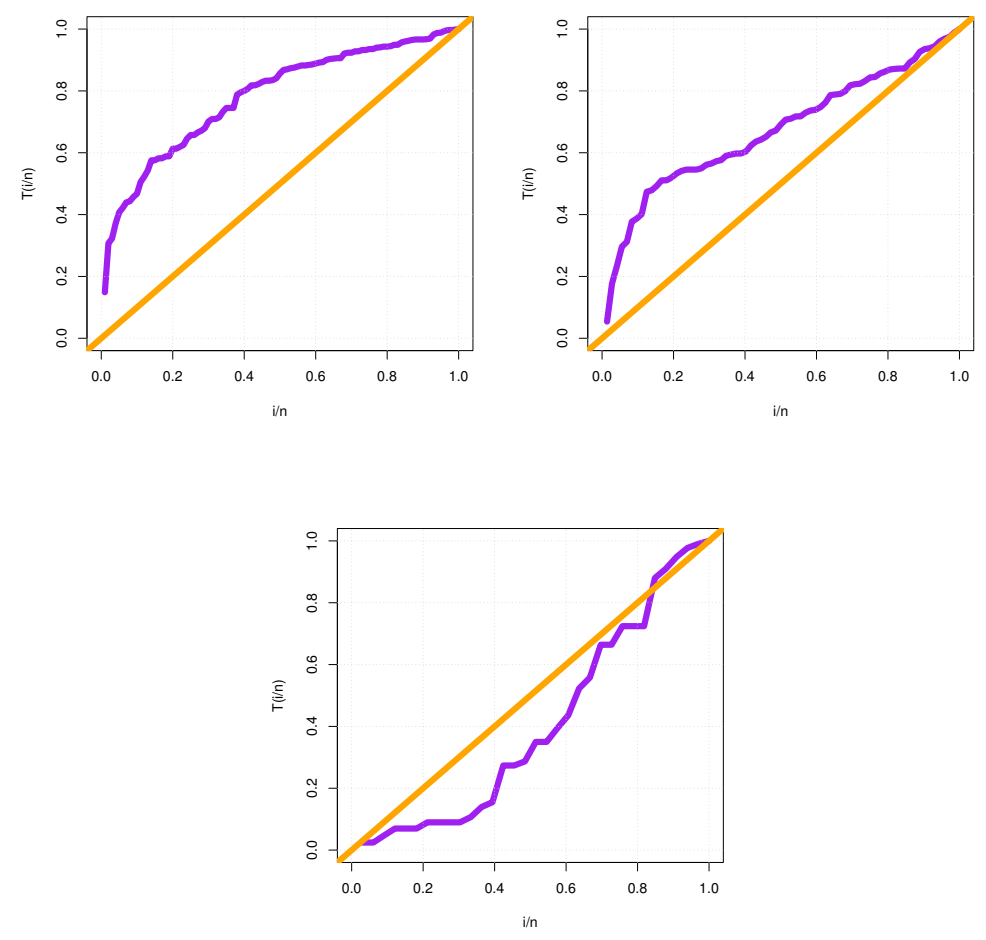

Figure 2: TTT plots.

and the score functions are obtained as follows

$$
\begin{aligned}
& \frac{\partial \mathcal{L}_{n}(\underline{\boldsymbol{\Phi}})}{\partial \beta}=\sum_{\varsigma=1}^{n} \nabla_{\varsigma}\left[\frac{1}{\beta}+\ln \left(1+z_{\varsigma}^{b}\right)\right]-a \sum_{\varsigma=1}^{n}\left(1+z_{\varsigma}^{b}\right)^{\beta} \ln \left(1+z_{\varsigma}^{b}\right) \\
& \frac{\partial \mathcal{L}_{n}(\underline{\boldsymbol{\Phi}})}{\partial b}=\sum_{\varsigma=1}^{n} \nabla_{\varsigma}\left[\frac{1}{b}+\frac{\left(1+\beta z_{\varsigma}^{b}\right) \ln z_{\varsigma}}{1+z_{\varsigma}^{b}}\right]-a \beta \sum_{\varsigma=1}^{n} z_{\varsigma}^{b} \ln z_{\varsigma}\left(1+z_{\varsigma}^{b}\right)^{\beta-1} \\
& \frac{\partial \mathcal{L}_{n}(\underline{\mathbf{\Phi}})}{\partial a}=\sum_{\varsigma=1}^{n} \frac{\nabla_{\varsigma}}{a}-\sum_{\varsigma=1}^{n}\left[\left(1+z_{\varsigma}^{b}\right)^{\beta}-1\right]
\end{aligned}
$$

Maximum likelihood estimators of the unknown parameters can be obtained using various techniques, such as software R, EM algorithm or Newton Raphson methods. In this section we conduct an important simulation study to consolidate our results. For this, 10, 000 censored samples (with sizes: $\left.n_{1}=15, n_{2}=25, n_{3}=50, n_{4}=130, n_{5}=350, n_{6}=500, n_{7}=1000\right)$ from EG-LL( $\left.\underline{\boldsymbol{\Phi}}\right)$ distribution are simulated. Simulated samples were generated with various parameters. Using R software and BB algorithm, the average values of the estimaotrs and their corresponding squred mean errors are given in Table 11. As shown in these results, the maximum likelihood estimators are convergent. 
Table 4: MLEs, SEs and CIs for the data set I

\begin{tabular}{|c|c|c|}
\hline Model & & Estimates \\
\hline \multirow[t]{3}{*}{$\operatorname{BXII}(\alpha, \beta)$} & MLEs & $5.941,0.187$ \\
\hline & SEs & $(1.279),(0.044)$ \\
\hline & CIs & $(3.43,8.45),(0.10,0.27)$ \\
\hline \multirow[t]{3}{*}{$\operatorname{MOBXII}(\alpha, \beta, \gamma)$} & MLEs & $1.192,4.834,838.73$ \\
\hline & SEs & $(0.952),(4.896),(229.34)$ \\
\hline & CIs & $(0,3.06),(0,14.43),(389.22,1288.24)$ \\
\hline \multirow[t]{3}{*}{$\operatorname{TLBXII}(\alpha, \beta, \gamma)$} & MLEs & $1.350,1.061,13.728$ \\
\hline & SEs & $(0.378),(0.384),(8.400)$ \\
\hline & CIs & $(0.61,2.09),(0.31,1.81),(0,30.19)$ \\
\hline \multirow[t]{3}{*}{$\operatorname{KwBXII}(\lambda, \theta, \alpha)}$, & MLEs & $48.103,79.516,0.351,2.730$ \\
\hline & SEs & (19.348), (58.186), (0.098), (1.077) \\
\hline & CIs & $(10.18,86.03),(0,193.56),(0.16,0.54),(0.62,4.84)$ \\
\hline \multirow[t]{3}{*}{$\operatorname{BBXII}(\lambda, \theta, \alpha)}$, & MLEs & $359.683,260.097,0.175,1.123$ \\
\hline & SEs & $(57.941),(132.213),(0.013),(0.243)$ \\
\hline & CIs & $(246.1,473.2),(0.96,519.2),(0.14,0.20),(0.65,1.6)$ \\
\hline \multirow[t]{3}{*}{$\operatorname{BEBXII}(\lambda, \theta, \alpha, \gamma)$} & MLEs & $0.381,11.949,0.937,33.402,1.705$ \\
\hline & SEs & $(0.078),(4.635),(0.267),(6.287),(0.478)$ \\
\hline & CIs & $(0.23,0.53),(2.86,21),(0.41,1.5),(21,45),(0.8,2.6)$ \\
\hline \multirow[t]{3}{*}{$\operatorname{FBBXII}(\lambda, \theta, \alpha, \beta, \gamma)$} & MLEs & $0.421,0.834,6.111,1.674,3.450$ \\
\hline & SEs & $(0.011),(0.943),(2.314),(0.226),(1.957)$ \\
\hline & CIs & $(0.4,0.44),(0.2 .7),(1.57,10.7),(1.23,2.1),(0,7)$ \\
\hline \multirow[t]{3}{*}{$\operatorname{FKwBXII}(\lambda, \theta, \alpha, \beta, \gamma)$} & MLEs & $0.542,4.223,5.313,0.411,4.152$ \\
\hline & SEs & $(0.137),(1.882),(2.318),(0.497),(1.995)$ \\
\hline & CIs & $(0.3,0.8),(0.53,7.9),(0.9,9),(0,1.7),(0.2,8)$ \\
\hline \multirow[t]{3}{*}{$\operatorname{ZBBXII}(\lambda, \alpha, \beta)$} & MLEs & $123.101,0.368,139.247$ \\
\hline & SEs & $(243.011),(0.343),(318.546)$ \\
\hline & CIs & $(0,599.40),(0,1.04),(0,763.59)$ \\
\hline \multirow[t]{3}{*}{$\operatorname{LL}(a)$} & MLEs & 1.629 \\
\hline & SEs & $(0.1288)$ \\
\hline & CIs & $(1.36,1.84)$ \\
\hline \multirow[t]{3}{*}{ E-LL } & MLEs & $5.221,2.319$ \\
\hline & SEs & $(0.5983),(0.1496)$ \\
\hline & CIs & $(4.2,6.2),(2,2.6)$ \\
\hline \multirow[t]{3}{*}{$\operatorname{WLL}(\beta, a)$} & MLEs & $1.116,0.71$ \\
\hline & SEs & $(6.5),(4.12)$ \\
\hline & CIs & $(0,16.1),(0,8.94)$ \\
\hline \multirow[t]{3}{*}{ 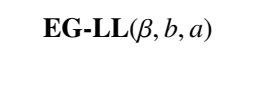 } & MLEs & $0.8076,3.314,0.0584$ \\
\hline & SEs & $(0.3504),(1.193),(0.025)$ \\
\hline & CIs & $(0.1,1.5),(0.9,5.7),(0.001,0.11)$ \\
\hline
\end{tabular}

\section{Modified Bagdonavičius-Nikulin validation test}

In this work, we are interested in the modified chi-squared type test proposed by Bagdonavičius and Nikulin (2011), Bagdonavičius et al. (2013) for parametric models with right censored data. Based on the maximum likelihood estimators of the non-grouped data, this test statistic is also based on the differences between the numbers of observed failures and the numbers of expected failures in the grouped intervals chosen. Thus, random grouping intervals are considered as data functions. The description of the construction for this chi-squared type test is developed in Voinov et al. (2013). The test statistic is defined as follows. Suppose that $Z_{1}, Z_{2}, \ldots, Z_{n}$ is a random sample with right censoring 
Table 5: $\mathrm{C}_{1}, \mathrm{C}_{2}, \mathrm{C}_{3}$ and $\mathrm{C}_{4}$ values for the data set $\mathbf{I}$

\begin{tabular}{cc}
\hline \hline Model & $\mathrm{C}_{1}, \mathrm{C}_{2}, \mathrm{C}_{3}, \mathrm{C}_{4}$ \\
\hline BXII & $382.94,388.15,383.06,385.05$ \\
MOBXII & $305.78,313.61,306.03,308.96$ \\
TLBXII & $323.52,331.35,323.77,326.70$ \\
KwBXII & $303.76,314.20,304.18,308.00$ \\
BBXII & $305.64,316.06,306.06,309.85$ \\
BEBXII & $305.82,318.84,306.46,311.09$ \\
FBBXII & $304.26,317.31,304.89,309.56$ \\
FKwBXII & $305.50,318.55,306.14,310.80$ \\
ZBBXII & $302.96,310.78,303.21,306.13$ \\
LL & $469.63,472.23,469.67,470.68$ \\
E-LL & $325.93,331.14,326.06,328.04$ \\
WLL & $510.69,515.91,510.82,512.84$ \\
EG-LL & $\mathbf{2 8 8 . 8 4 , 2 9 6 . 6 6 , 2 8 9 . 0 9 , 2 9 2 . 0 0}$ \\
\hline \hline
\end{tabular}

Table 6: MLEs, SEs and CIs for the data set II

\begin{tabular}{|c|c|c|}
\hline Model & & Estimates \\
\hline \multirow{4}{*}{$\operatorname{BXII}(\alpha, \beta)$} & MLEs & $3.102,0.465$, \\
\hline & SEs & $(0.538),(0.077)$ \\
\hline & CIs & $(2.05,4.16),(0.31,0.62)$ \\
\hline & MLEs & $2.259,1.533,6.760$ \\
\hline \multirow{3}{*}{$\operatorname{MOBXII}(\alpha, \beta, \gamma)$} & SEs & $(0.864),(0.907),(4.587)$ \\
\hline & CIs & $(0.57,3.95),(0,3.31),(0,15.75)$ \\
\hline & MLEs & $2.393,0.458,1.796$ \\
\hline \multirow{3}{*}{$\operatorname{TLBXII}(\alpha, \beta, \gamma)$} & SEs & $(0.907),(0.244),(0.915)$ \\
\hline & CIs & $(0.62,4.17),(0,0.94),(0.002,3.59)$ \\
\hline & MLEs & $14.105,7.424,0.525,2.274$ \\
\hline \multirow[t]{3}{*}{$\operatorname{KwBXII}(\lambda, \theta, \alpha, \beta)$} & SEs & $(10.805),(11.850),(0.279),(0.990)$ \\
\hline & CIs & $(0,35.28),(0.30 .65),(0,1.07),(0.33,4.21)$ \\
\hline & MLEs & $2.555,6.058,1.800,0.294$ \\
\hline \multirow[t]{3}{*}{$\operatorname{BBXII}(\lambda, \theta, \alpha, \beta)$} & SEs & $(1.859),(10.391),(0.955),(0.466)$ \\
\hline & CIs & $(0,6.28),(0,26.42),(0,3.67),(0,1.21)$ \\
\hline & MLEs & $1.876,2.991,1.780,1.341,0.572$ \\
\hline \multirow[t]{3}{*}{$\operatorname{BEBXII}(\lambda, \theta, \alpha, \beta, \gamma)$} & SEs & $(0.094),(1.731),(0.702),(0.816),(0.325)$ \\
\hline & CIs & $(1.7,2.06),(0,6.4),(0.40,3.2),(0,2.9),(0,1.21)$ \\
\hline & MLEs & $0.621,0.549,3.838,1.381,1.665$ \\
\hline \multirow[t]{3}{*}{$\operatorname{FBBXII}(\lambda, \theta, \alpha, \beta, \gamma)$} & SEs & $(0.541),(1.011),(2.785),(2.312),(0.436)$ \\
\hline & CIs & $(0,1.7),(0,2.5),(0,9.3),(0,5.9),(0.8,4.5)$ \\
\hline & MLEs & $0.558,0.308,3.999,2.131,1.475$ \\
\hline \multirow{3}{*}{$\operatorname{FKwBXII}(\lambda, \theta, \alpha, \beta, \gamma)$} & SEs & $(0.442),(0.314),(2.082),(1.833),(0.361)$ \\
\hline & CIs & $(0,1.4),(0,0.9),(0,3.1),(0,5.7),(0.76,2.2)$ \\
\hline & MLEs & 2.275 \\
\hline \multirow[t]{3}{*}{$\operatorname{LL}(a)$} & SEs & $(0.223)$ \\
\hline & CIs & $(1.9,2.7)$ \\
\hline & MLEs & $1.951,2.25$ \\
\hline \multirow[t]{3}{*}{ E-LL } & SEs & $(0.229),(0.2069)$ \\
\hline & CIs & $(1.5,2.3),(2.1,2.9)$ \\
\hline & MLEs & $0.785,1.254$ \\
\hline \multirow[t]{3}{*}{$\operatorname{WLL}(\beta, a)$} & SEs & $(0.00),(0.00)$ \\
\hline & CIs &,-- \\
\hline & MLEs & $0.3706,2.9555,0.9115$ \\
\hline \multirow{2}{*}{$\mathbf{E G - L L}(\beta, b, a)$} & SEs & $(0.1376),(0.581),(0.6984)$ \\
\hline & CIs & $(0.14,0.66),(1.7,4.1),(0,2.3)$ \\
\hline
\end{tabular}


Table 7: $\mathrm{C}_{1}, \mathrm{C}_{2}, \mathrm{C}_{3}$ and $\mathrm{C}_{4}$ values for the data set II

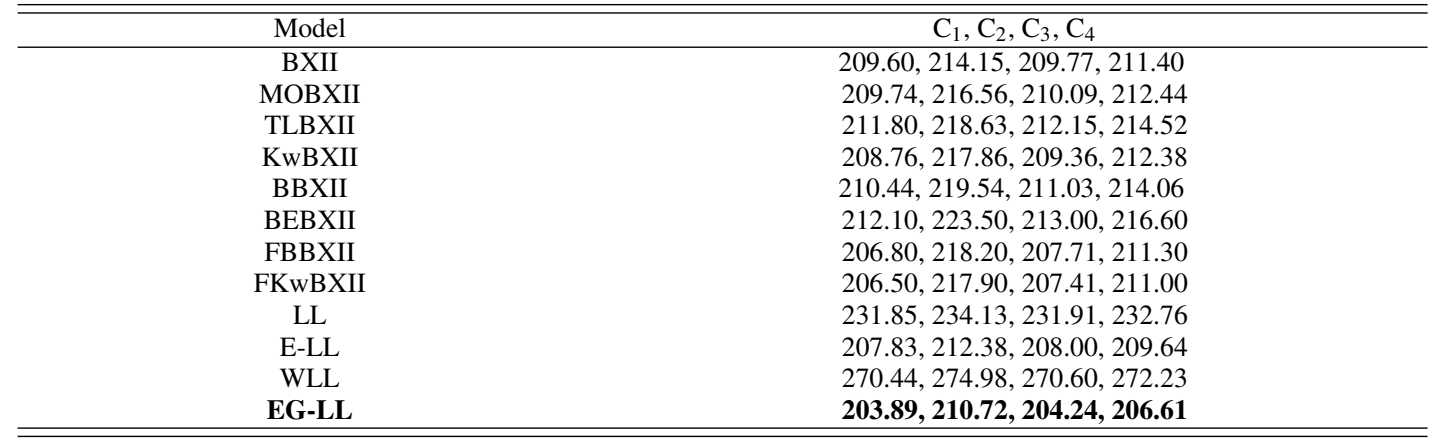
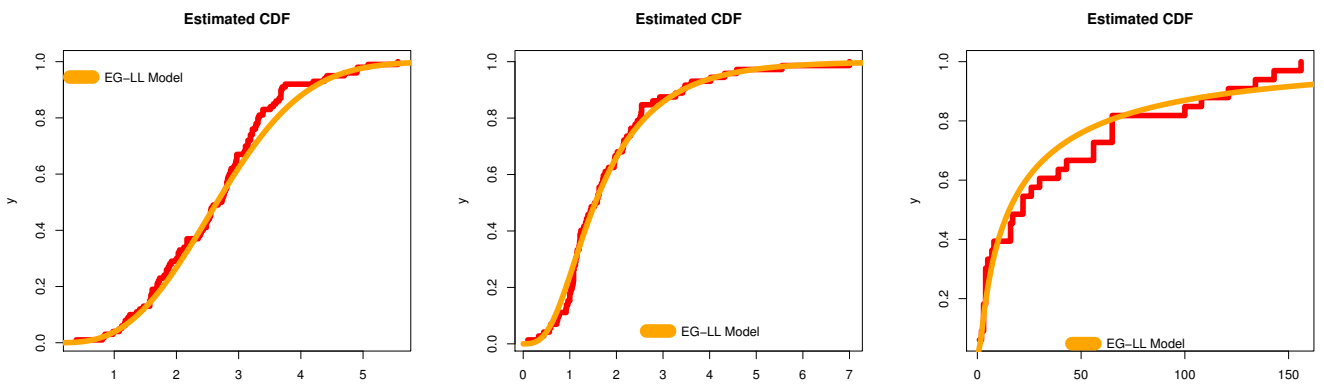

Figure 3: The estimated CDFs.
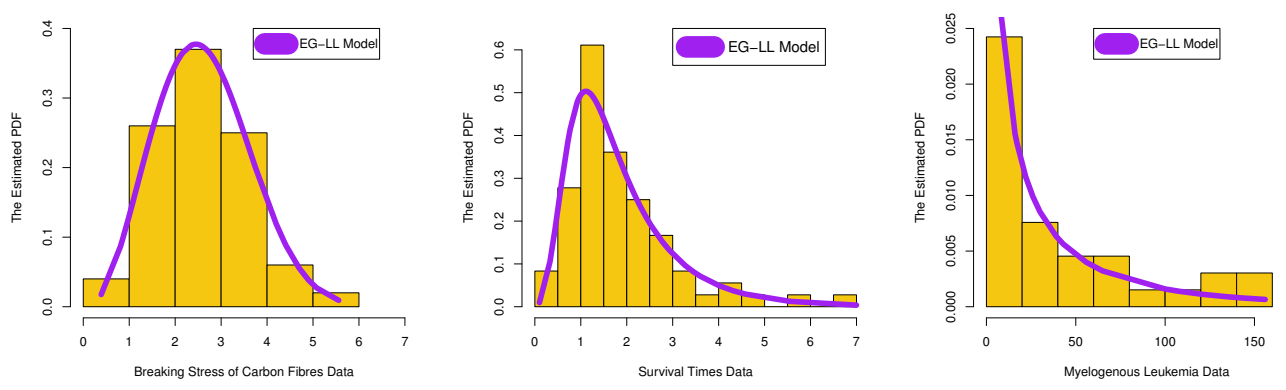

Figure 4: The estimated PDFs.

from a parametric model and a finite time $\tau$. The test statistic is defined as

$$
Y_{n}^{2}=\sum_{j=1}^{n} \frac{\left(\mathbf{U}_{j}-e_{j}\right)^{2}}{\mathbf{U}_{j}}+\mathbf{Q}
$$


Table 8: MLEs, SEs and CIs for the data set III

\begin{tabular}{|c|c|c|}
\hline Model & & Estimates \\
\hline \multirow[t]{3}{*}{$\overline{\mathrm{BXII}}(\alpha, \beta)$} & MLEs & $58.711,0.006$ \\
\hline & SEs & $(42.382),(0.004)$ \\
\hline & CIs & $(0,141.78),(0,0.01)$ \\
\hline \multirow{3}{*}{$\operatorname{MOBXII}(\alpha, \beta, \gamma)$} & MLEs & $11.838,0.078,12.251$ \\
\hline & SEs & (4.368), (0.013), (7.770) \\
\hline & CIs & $(0,141.78),(0,0.01),(0,27.48)$ \\
\hline \multirow{3}{*}{$\operatorname{TLBXII}(\alpha, \beta, \gamma)$} & MLEs & $0.281,1.882,50.215$ \\
\hline & SEs & $(0.288),(2.402),(176.50)$ \\
\hline & CIs & $(0,0.85),(0,6.59),(0,396.16)$ \\
\hline \multirow[t]{3}{*}{$\operatorname{KwBXII}(\lambda, \theta, \alpha, \beta)$} & MLEs & $9.201,36.428,0.242,0.941$ \\
\hline & SEs & $(10.060),(35.650),(0.167),(1.045)$ \\
\hline & CIs & $(0,28.912),(0,106.30),(0,0.57),(0,2.99)$ \\
\hline \multirow{3}{*}{$\operatorname{BBXII}(\lambda, \theta, \alpha, \beta)$} & MLEs & $96.104,52.121,0.104,1.227$ \\
\hline & SEs & (41.201), (33.490), (0.023), (0.326) \\
\hline & CIs & $(15.4,176.8),(0,117.8),(0.6,0.15),(0.59,1.9)$ \\
\hline \multirow{3}{*}{$\operatorname{BEBXII}(\lambda, \theta, \alpha, \beta, \gamma)$} & MLEs & $0.087,5.007,1.561,31.270,0.318$ \\
\hline & SEs & $(0.077),(3.851),(0.012),(12.940),(0.034)$ \\
\hline & CIs & $(0,0.3),(0,12.6),(1.5,1.6),(5.9,56.6),(0.3,0.4)$ \\
\hline \multirow[t]{3}{*}{$\operatorname{FBBXII}(\lambda, \theta, \alpha, \beta, \gamma)$} & MLEs & $15.194,32.048,0.233,0.581,21.855$ \\
\hline & SEs & (11.58), (9.867), (0.091), (0.067), (35.548) \\
\hline & CIs & $(0,37.8),(12.7,51.4),(0.05,0.4),(0.45,0.7),(0,91.5)$ \\
\hline \multirow[t]{3}{*}{$\operatorname{FKwBXII}(\lambda, \theta, \alpha, \beta, \gamma)$} & MLEs & $14.732,15.285,0.293,0.839,0.034$ \\
\hline & SEs & $(12.390),(18.868),(0.215),(0.854),(0.075)$ \\
\hline & CIs & $(0,39.02),(0,52.27),(0,0.71),(0,2.51),(0,0.18)$ \\
\hline \multirow[t]{3}{*}{$\operatorname{ZBBXII}(\lambda, \alpha, \beta, \gamma)$} & MLEs & $41.973,0.157,44.263$ \\
\hline & SEs & $(38.787),(0.082),(47.648)$ \\
\hline & CIs & $(0,117.99),(0,0.32),(0,137.65)$ \\
\hline \multirow[t]{3}{*}{$\operatorname{LL}(a)$} & MLEs & 0.507 \\
\hline & SEs & $(0.071)$ \\
\hline & CIs & $(0.36,0.64)$ \\
\hline \multirow[t]{3}{*}{ E-LL } & MLEs & $5.594,0.7647$ \\
\hline & SEs & (1.1834), (0.093) \\
\hline & CIs & $(3.1,7.9),(0.52,0.88)$ \\
\hline \multirow{3}{*}{$\operatorname{WLL}(\beta, a)$} & MLEs & $1.069,0.224$ \\
\hline & SEs & $(0.00),(0.00)$ \\
\hline & CIs &,-- \\
\hline \multirow[t]{3}{*}{$\mathbf{E G - L L}(\beta, b, a)$} & MLEs & $0.1269,4.78,0.141$ \\
\hline & SEs & $(0.046),(1.32),(0.090)$ \\
\hline & CIs & $(0.02,0.22),(2.16,7.44),(0,0.32)$ \\
\hline
\end{tabular}

where $\mathbf{U}_{j}$ and $e_{j}$ are the observed and the expected numbers of failure in grouping intervals, and $\mathbf{Q}$ is

$$
\begin{aligned}
\mathbf{Q} & =\mathbf{W}^{T} \hat{\mathbf{G}}^{-} \mathbf{W}, \\
\hat{\mathbf{W}} & =\hat{\mathbf{C}} \hat{\mathbf{A}}^{-1} \mathbf{T}=\left(\hat{\mathbf{W}}_{1}, \ldots, \hat{\mathbf{W}}_{s}\right)^{T}, \\
\mathbf{T}_{j} & =\frac{1}{\sqrt{n}}\left(\mathbf{U}_{j}-e_{j}\right) \\
\mathbf{W} & =\sum_{j=1}^{r} \hat{\mathbf{C}}_{j} \hat{\mathbf{A}}_{j}^{-1} \mathbf{T}_{j}, \\
\hat{\mathbf{G}} & =\left[\hat{g}^{\prime}\right]_{s \times s}, \\
\hat{g}^{\prime} & =\hat{\imath}^{\prime}-\left.\sum_{j=1}^{r} \hat{\mathbf{C}}_{j} \hat{\mathbf{C}}_{j} \hat{\mathbf{A}}_{j}^{-1}\right|_{(S=1, \ldots, n, j=1, \ldots, r \text { and },,=1, \ldots, s)} .
\end{aligned}
$$


Table 9: $C_{1}, C_{2}, C_{3}$ and $C_{4}$ values for the data set III

\begin{tabular}{|c|c|}
\hline Model & $\mathrm{C}_{1}, \mathrm{C}_{2}, \mathrm{C}_{3}, \mathrm{C}_{4}$ \\
\hline BXII & $328.20,331.19,328.60,329.19$ \\
\hline MOBXII & $315.54,320.01,316.37,317.04$ \\
\hline TLBXII & $316.26,320.73,317.09,317.76$ \\
\hline KwBXII & $317.36,323.30,318.79,319.34$ \\
\hline BBXII & $316.46,322.45,317.89,318.47$ \\
\hline BEBXII & $317.58,325.06,319.80,320.09$ \\
\hline FBBXII & $317.86,325.34,320.08,320.36$ \\
\hline FKwBXII & $317.76,325.21,319.98,320.26$ \\
\hline ZBBXII & $313.86,318.35,314.39,315.36$ \\
\hline WLL & $378.82,381.79,379.27,379.84$ \\
\hline LL & $362.71,364.20,362.83,363.21$ \\
\hline E-LL & $315.46,318.45,315.85,316.47$ \\
\hline EG-LL & $309.84,314.33,310.67,311.35$ \\
\hline
\end{tabular}
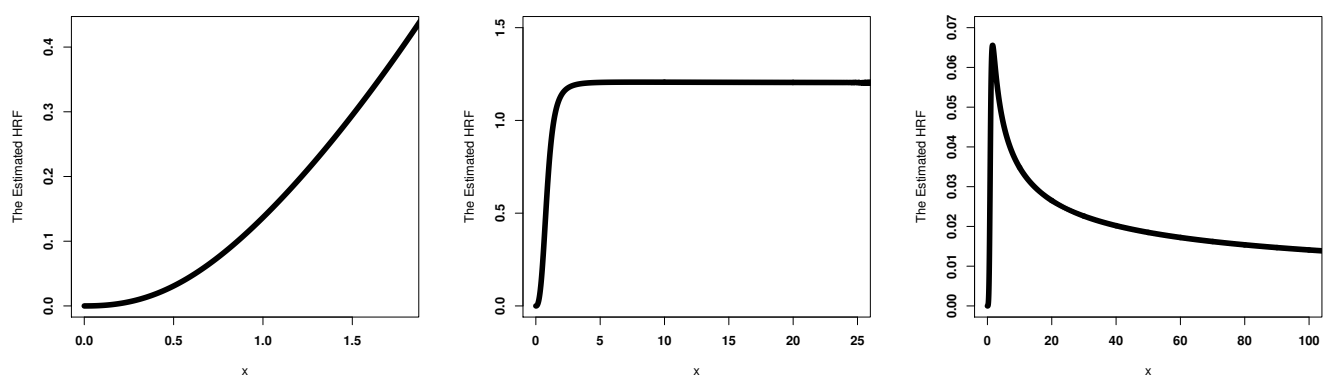

Figure 5: The estimated HRFs.
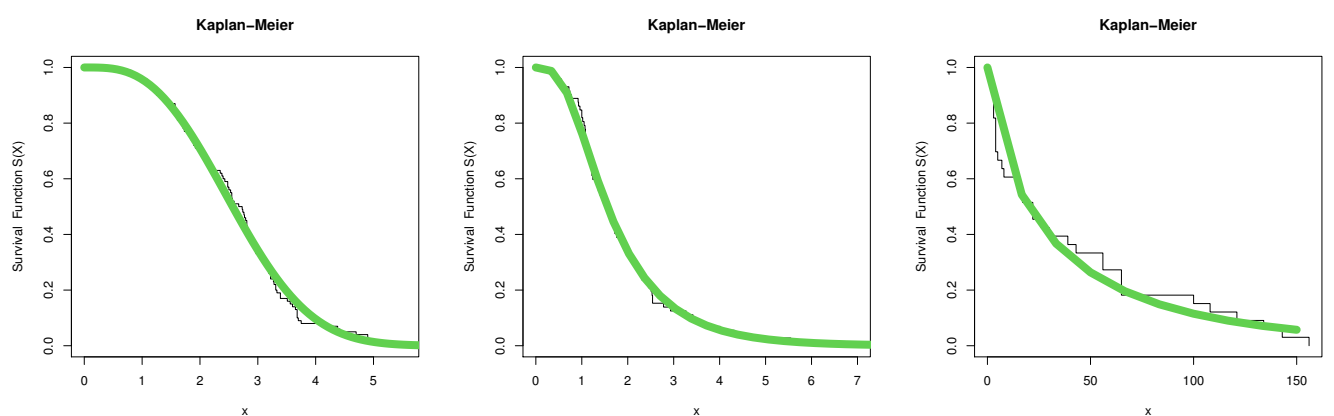

Figure 6: The Kaplan-Meier survival plots.

The limits $\rho_{j}$ of $r$ random grouping intervals $\varsigma_{j}=\left[\rho_{j-1}, \rho_{j}\right.$ [ are chosen so that the expected failure times falling into these intervals are the same for each $\left.j\right|_{(j=1, \ldots, r-1)}$ and

$$
\hat{\rho}_{r}=\max \left(z_{()}, \tau\right)
$$



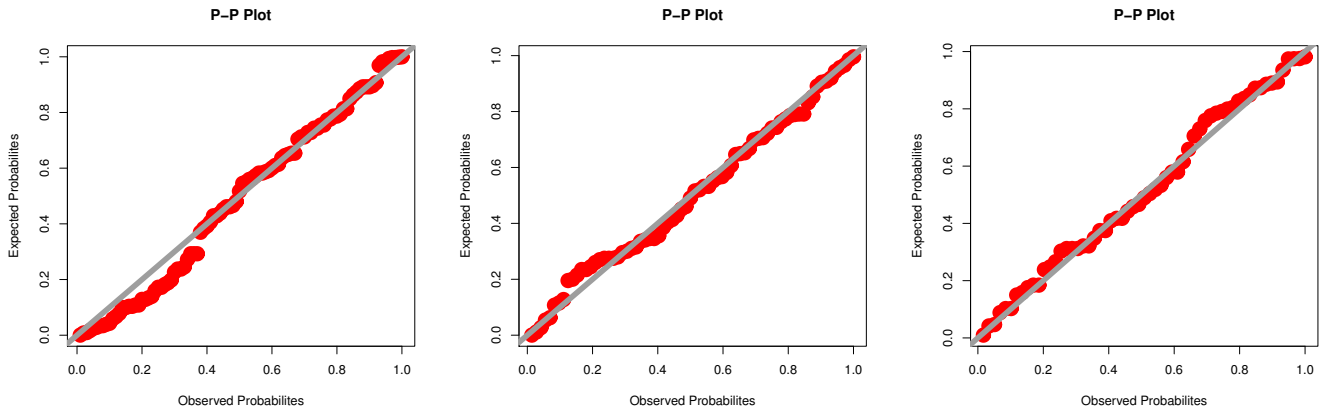

Figure 7: The PP plots.

Table 10: MLEs for $\hat{\beta}, \hat{a}, \hat{b}$ and their MSEs

\begin{tabular}{lllllll}
\hline \hline$N=10.000$ & $\hat{\beta}=2$ & MSE & $\hat{a}=0.8$ & MSE & $\hat{b}=2.5$ & MSE \\
\hline$n_{1}=15$ & 1.9623 & 0.0072 & 0.7734 & 0.0068 & 2.5163 & 0.0086 \\
$n_{2}=25$ & 1.9684 & 0.0067 & 0.7796 & 0.0058 & 2.5142 & 0.0072 \\
$n_{3}=50$ & 1.9736 & 0.0046 & 0.7812 & 0.0049 & 2.5106 & 0.0059 \\
$n_{4}=130$ & 1.9794 & 0.0038 & 0.7876 & 0.0032 & 2.5094 & 0.0042 \\
$n_{5}=350$ & 1.9878 & 0.0027 & 0.7912 & 0.0024 & 2.5057 & 0.0034 \\
$n_{6}=500$ & 1.9945 & 0.0019 & 0.7998 & 0.0015 & 2.5023 & 0.0027 \\
$n_{7}=1000$ & 1.9992 & 0.0012 & 0.8006 & 0.0009 & 2.5007 & 0.0015 \\
\hline & $\hat{\beta}=1$ & MSE & $\hat{a}=1.5$ & MSE & $\hat{b}=1.3$ & MSE \\
\hline$n_{1}=15$ & 1.0463 & 0.0086 & 1.5236 & 0.0092 & 1.2624 & 0.0079 \\
$n_{2}=25$ & 1.0425 & 0.0073 & 1.5194 & 0.0083 & 1.2694 & 0.0068 \\
$n_{3}=50$ & 1.0362 & 0.0065 & 1.5137 & 0.0071 & 1.2732 & 0.0057 \\
$n_{4}=130$ & 1.0247 & 0.0052 & 1.5092 & 0.0062 & 1.2876 & 0.0043 \\
$n_{5}=350$ & 1.0123 & 0.0037 & 1.5067 & 0.0048 & 1.2943 & 0.0032 \\
$n_{6}=500$ & 1.0067 & 0.0022 & 1.5034 & 0.0036 & 1.2976 & 0.0017 \\
$n_{7}=1000$ & 1.0011 & 0.0010 & 1.5002 & 0.0012 & 1.3006 & 0.0008 \\
\hline & $\hat{\beta}=0.7$ & MSE & $\hat{a}=3$ & MSE & $\hat{b}=0.6$ & MSE \\
\hline$n_{1}=15$ & 0.7234 & 0.0098 & 2.9516 & 0.0103 & 0.5764 & 0.0109 \\
$n_{2}=25$ & 0.7189 & 0.0079 & 2.9634 & 0.0089 & 0.5823 & 0.0092 \\
$n_{3}=50$ & 0.7126 & 0.0061 & 2.9718 & 0.0077 & 0.5886 & 0.0078 \\
$n_{4}=130$ & 0.7079 & 0.0047 & 2.9834 & 0.0051 & 0.5916 & 0.0059 \\
$n_{5}=350$ & 0.7065 & 0.0034 & 2.9927 & 0.0034 & 0.5943 & 0.0043 \\
$n_{6}=500$ & 0.7027 & 0.0023 & 2.9976 & 0.0027 & 0.5986 & 0.0028 \\
$n_{7}=1000$ & 0.7009 & 0.0010 & 3.0002 & 0.0018 & 0.6004 & 0.0013 \\
\hline \hline
\end{tabular}

The estimated $\hat{\rho}_{j}$ is defined by

$$
\hat{\rho}_{j}=\left.H^{-1}\left(\frac{E_{j}-\sum_{=1}^{\varsigma^{-1}} H_{\underline{\Phi}}(z)}{n-\varsigma+1}, \underline{\mathbf{\Phi}}\right)\right|_{\left(\hat{\rho}_{k}=\max \left(z_{(n)} \tau\right)\right)}
$$

where $H_{\Phi}(z)$ is the cumulative hazard function $(\mathrm{CHRF})$ of the model distribution. This test statistic $Y_{n}^{2}$ follows a chi-squared distribution. For more details see Goual et al. (2019), Ibrahim et al. (2019, 2020), Yadav et al. (2020), Mansour et al. (2020a,b), Salah et al. (2020), Goual and Yousof (2020) and Yousof et al. (2021). 

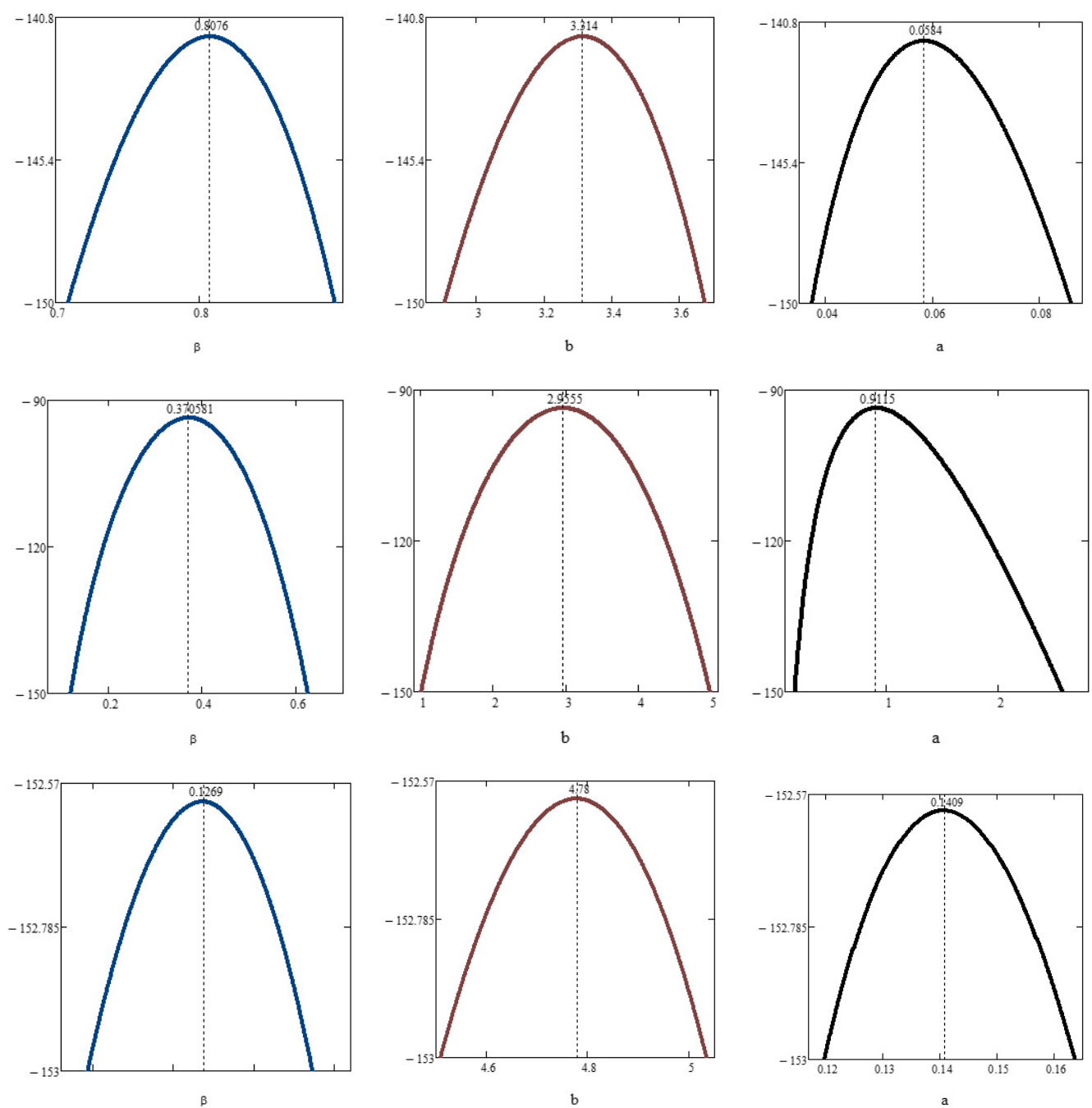

Figure 8: Profile likelihood plots.

\subsection{Choice of random grouping intervals}

Suppose that $Z_{1}, Z_{2}, \ldots, Z_{n}$ is a random sample with right censoring from EG-LL( $\left.\underline{\boldsymbol{\Phi}}\right)$ distribution and a finite time $\tau$. In our case, the estimated $\hat{\rho}_{j}$ is obtained as follows

$$
\hat{\rho}_{j}=\left[\left(\frac{E_{j}-\sum_{=1}^{S^{-1}} H_{\underline{\Phi}}(z)}{\hat{a}(n-\varsigma+1)}+1\right)^{1 / \hat{\beta}}-1\right]^{1 / \hat{b}},
$$

where $\boldsymbol{\Phi}=(\hat{\beta}, \hat{a}, \hat{b})^{T}$ are the maximum likelihood estimators of the unknown parameters $\underline{\Phi}=$ $(\beta, a, b)^{\frac{T}{T}}$ of the initial data, and $H_{\underline{\Phi}}(z)$ is the cumulative HRF of the EG-LL( $(\underline{\Phi})$ distribution. 


\subsection{Quadratic form $\mathbf{Q}$}

To calculate the quadratic form $\mathbf{Q}$ of the statistic $Y_{n}^{2}$, and since its distribution does not depend on the parameters, we can use the estimated matrices $\hat{\mathbf{W}}, \hat{\mathbf{C}}$ and the estimated information matrix $\hat{\mathbf{I}}$. The elements of $\hat{\mathbf{C}}$ are

$$
\hat{\mathbf{C}}_{j}=\frac{1}{n} \sum_{\varsigma: z_{\zeta} \in \varsigma_{j}}^{n} \nabla_{\varsigma} \frac{\partial}{\partial \underline{\Phi}} \ln h_{\underline{\Phi}}\left(z_{\varsigma}\right)
$$

and are obtained as shown below

$$
\begin{aligned}
& \hat{\mathbf{C}}_{1 j}=\frac{1}{n} \sum_{\varsigma: z_{\varsigma} \in \varsigma_{j}}^{n} \nabla_{\varsigma}\left[\frac{1}{\beta}+\ln \left(1+z_{\varsigma}^{b}\right)\right], \\
& \hat{\mathbf{C}}_{2 j}=\frac{1}{n} \sum_{\varsigma: z_{\varsigma} \in \varsigma_{j}}^{n} \nabla_{\varsigma}\left[\frac{1}{b}+\frac{\left(1+\beta z_{\varsigma}^{b}\right) \ln z_{\varsigma}}{1+z_{\varsigma}^{b}}\right], \\
& \hat{\mathbf{C}}_{3 j}=\frac{1}{n} \sum_{\varsigma: z_{\varsigma} \in \epsilon_{j}}^{n} \frac{\nabla_{\varsigma}}{a} .
\end{aligned}
$$

Therefore, the estimated matrix $\hat{\mathbf{W}}$ can be deduced from $\hat{\mathbf{C}}$.

\subsection{Estimated information matrix $\hat{\mathbf{I}}$}

We also need the information matrix $\hat{\mathbf{I}}$ of the $\operatorname{EG}-\operatorname{LL}(\underline{\Phi})$ distribution with right censoring. The elements of the matrix can be obtained as follows,

$$
\begin{aligned}
& \hat{\imath}_{11}=\frac{1}{n} \sum_{\varsigma=1}^{n} \nabla_{\varsigma}\left(\frac{1}{\beta}+\ln \left(1+z_{\varsigma}^{b}\right)\right)^{2}, \\
& \hat{\imath}_{22}=\frac{1}{n} \sum_{\varsigma=1}^{n} \nabla_{\varsigma}\left(\frac{1}{b}+\frac{\left(1+\beta z_{\varsigma}^{b}\right) \ln z_{\varsigma}}{1+z_{\varsigma}^{b}}\right)^{2}, \\
& \hat{\imath}_{33}=\frac{1}{n} \sum_{\varsigma=1}^{n} \frac{\nabla_{\varsigma}}{a^{2}}, \\
& \hat{\imath}_{12}=\frac{1}{n} \sum_{\varsigma=1}^{n} \nabla_{\varsigma}\left(\frac{1}{\beta}+\ln \left(1+z_{\varsigma}^{b}\right)\right)\left(\frac{1}{b}+\frac{\left(1+\beta z_{\varsigma}^{b}\right) \ln z_{\varsigma}}{1+z_{\varsigma}^{b}}\right), \\
& \hat{\imath}_{13}=\frac{1}{n} \sum_{\varsigma=1}^{n} \frac{\nabla_{\varsigma}}{a}\left(\frac{1}{\beta}+\ln \left(1+z_{\varsigma}^{b}\right)\right), \\
& \hat{\imath}_{23}=\frac{1}{n} \sum_{\varsigma=1}^{n} \frac{\nabla_{\varsigma}}{a}\left(\frac{1}{b}+\frac{\left(1+\beta z_{\varsigma}^{b}\right) \ln z_{\varsigma}}{1+z_{\varsigma}^{b}}\right)
\end{aligned}
$$

As all the components of the statistic are given explicitly, then the test statistic for the $\operatorname{EG}-\operatorname{LL}(\underline{\mathbf{\Phi}})$ distribution with unknown parameters and right censored data are obtained. This statistic follows a 
Table 11: Simulated levels of significance for $Y_{n}^{2}(\underline{\Phi})$ test under the EG-LL model against their theoretical values $(\varepsilon 0.01,0.05,0.10)$

\begin{tabular}{llllllll}
\hline \hline$N=10,000$ & $n=15$ & $n=25$ & $n=50$ & $n=130$ & $n=350$ & $n=500$ & $n=1000$ \\
\hline$\varepsilon=1 \%$ & 0.0065 & 0.0078 & 0.0082 & 0.0085 & 0.0092 & 0.0098 & 0.0103 \\
$\varepsilon=5 \%$ & 0.0413 & 0.0445 & 0.0462 & 0.0475 & 0.0481 & 0.0497 & 0.0502 \\
$\varepsilon=10 \%$ & 0.0937 & 0.0959 & 0.0965 & 0.0979 & 0.0983 & 0.0998 & 0.1004 \\
\hline \hline
\end{tabular}

chi-squared distribution with $r$ degrees of freedom.

$$
Y_{n}^{2}(\underline{\Phi})=\sum_{j=1}^{r} \frac{\left(\mathbf{U}_{j}-e_{j}\right)^{2}}{\mathbf{U}_{j}}+\hat{\mathbf{W}}^{T} \mathbf{M}^{-1} \hat{\mathbf{W}},
$$

where

$$
\mathbf{M}^{-1}=\left(\hat{\imath},-\sum_{j=1}^{r} \hat{\mathbf{C}}_{j} \hat{\mathbf{C}}_{j} \hat{\mathbf{A}}_{j}^{-1}\right)^{-1} .
$$

\subsection{Simulated samples (right censored case)}

For testing the null hypothesis $\mathbb{H}_{0}$ right censored data that arises from the EG-LL model, we compute the criteria statistic $Y_{n}^{2}(\underline{\boldsymbol{\Phi}})$ as defined above for 10,000 simulated samples from the hypothesized distribution with different $\operatorname{sizes}(n=15,25,50,130,350,500,1000)$. Then, we calculate empirical levels of significance, when $Y>\chi_{\varepsilon}^{2}(r)$ correponds to theoretical levels of significance $(\varepsilon=0.10,0.05,0.01)$, and where $r=5$. The results are reported in Table 11. The null hypothesis $\mathbb{H}_{0}$ for which simulated samples are fitted by the EG-LL distribution, is widely validated for the different levels of significance. Therefore, the test proposed in this work, can be used to fit data from this new distribution.

\section{Data analysis (right censored case)}

We have analyzed a lymphoma data set consisting of times (in months) from diagnosis to death for 31 individuals with advanced non Hodgkin's lymphoma clinical symptoms, using our model. This data has been analyzed by Gijbels and Gurler (2003) using the exponential change point model. Among these 31 observations, 11 of the times are censored, because the patients were alive at the last time of follow-up.

2.5, 4.1, 4.6, 6.4, 6.7, 7.4, 7.6, 7.7, 7.8, 8.8, 13.3, 13.4, 18.3, 19.7, 21.9, 24.7, 27.5, 29.7, 30.1*, $32.9,33.5,35.4^{*}, 37.7^{*}, 40.9^{*}, 42.6^{*}, 45.4^{*}, 48.5^{*}, 48.9^{*}, 60.4^{*}, 64.4^{*}, 66.4^{*}$, where $*$ denotes a censored observation. We use the test statistic provided above to verify if data is modeled by EG-LL distribution and to that end, we first calculate the maximum likelihood estimators of the unknown parameters

$$
\underline{\Phi}=(\hat{\beta}, \hat{b}, \hat{a})^{T}=(0.8469,1.486,2.9568)^{T} .
$$

Data are grouped into $r=5$ intervals $\varsigma_{j}$. We provide the necessary calculus in the following Table 12 . Then, we obtain the value of the test statistic $Y_{n}^{2}$,

$$
Y_{n}^{2}=X^{2}+\mathbf{Q}=4.9563+3.8496=8.8059 .
$$


Table 12: values of $\hat{\rho}_{j}, e_{j}, \mathbf{U}_{j}, \hat{\mathbf{C}}_{1 j}, \hat{\mathbf{C}}_{2 j}$ and $\hat{\mathbf{C}}_{3 j}$

\begin{tabular}{llllll}
\hline \hline$\hat{\boldsymbol{\rho}}_{j}$ & 7.2 & 8.5 & 15.9 & 31.5 & 66.4 \\
\hline $\mathbf{U}_{J}$ & 5 & 4 & 3 & 7 & 12 \\
$\hat{\mathbf{C}}_{1 j}$ & 1.3648 & 1.6342 & 1.2131 & 0.9362 & 0.8376 \\
$\hat{\mathbf{C}}_{2 j}$ & 0.9881 & 0.8734 & 0.7823 & 1.0234 & 0.9236 \\
$\hat{\mathbf{C}}_{3 j}$ & 1.6910 & 1.3528 & 1.0146 & 2.0292 & 0.6764 \\
$e_{j}$ & 2.1836 & 2.1836 & 2.1836 & 2.1836 & 2.1836 \\
\hline \hline
\end{tabular}

Table 13: $Y_{n}^{2}$ for all models

\begin{tabular}{ll}
\hline \hline Model & $Y_{n}^{2}$ \\
\hline EG-LL & $\mathbf{8 . 8 0 5 9}$ \\
MOBXII & 9.1748 \\
TLBXII & 9.3021 \\
KwBXII & 9.4536 \\
BEBXII & 9.6748 \\
BBXII & 9.3214 \\
FKwBXII & 9.8695 \\
ZBBXII & 9.0345 \\
WLL & 11.054 \\
LL & 10.956 \\
E-LL & 9.1356 \\
FBBXII & 10.075 \\
BXII & 10.265 \\
\hline \hline
\end{tabular}

For significance level $\varepsilon=0.05$, the critical value $\chi_{5}^{2}=11.0705$ is greater than the value of $Y_{n}^{2}=$ 8.8059. Thus, we can say that the proposed model EG-LL fit the data. We also calculated the test statistic $Y_{n}^{2}$ to fit the data to the competing models. The results are given in Table 14. Table 14 shows that the EG-LL has the lowest value of $Y_{n}^{2}$ among all the other competitive models.

\section{Conclusions}

In this paper, we extended the log-logistic (LL) model by proposing and studying a new probability distribution called the exponential generalized log-logistic (EG-LL) model. Different non-Bayesian estimation methods under complete model scheme are considered such as the maximum likelihood estimation, the Anderson Darling estimation, the ordinary least square estimation, the Cramér-vonMises estimation, the weighted least square estimation, the left tail-Anderson Darling estimation, and the right tail Anderson Darling estimation methods. Numerical simulation studies were performed for comparing these estimation methods using different sample sizes and three different combinations of parameters. The potentiality of the EG-LL model is illustrated using three real data sets and the model is compared with many other well-known generalizations. The new model was proven worthy in modeling breaking stress, survival times and medical data sets. The Barzilai-Borwein algorithm is employed via a simulation study for assessing the performance of the estimators with different sample sizes as sample size tends to $\infty$. Using the Bagdonavičius-Nikulin goodness-of-fit test for validation, we propose a modified chi-square GOF tests for the EG-LL model. We have analyzed a lymphoma data set consisting of times (in months) from diagnosis to death for 31 individuals with advanced non Hodgkin's lymphoma clinical symptoms by using our model under the modified Bagdonavičius-Nikulin goodness-of-fit test statistic. Based on the MLEs, the modified Bagdonavičius-Nikulin goodness-of-fit test recovered the loss of information for the grouping data and fol- 
lows the chi-square distribution. The corresponding elements of the modified Bagdonavičius-Nikulin goodness-of-fit criteria tests are, therefore, explicitly derived.

\section{Acknowledgment}

The authors gratefully acknowledge the very thoughtful and constructive comments and suggestions of the Editor-in-Chief and the two reviewers which resulted in much improved paper.

\section{References}

Aarset MV (1987). How to identify a bathtub hazard rate, IEEE Transactions on Reliability, 36, $106-108$.

Abouelmagd THM, Hamed MS, Almamy JA, Ali MM, Yousof HM, and Korkmaz MC (2019). Extended Weibull log-logistic distribution, Journal of Nonlinear Sciences and Applications, 12, 523-534.

Altun E, Yousof HM, and Hamedani GG (2018 a). A new log-location regression model with influence diagnostics and residual analysis, Facta Universitatis, Series: Mathematics and Informatics, 33, 417-449.

Altun E, Yousof HM, Chakraborty S, and Handique L (2018b). Zografos-Balakrishnan Burr XII distribution: regression modeling and applications, International Journal of Mathematics and Statistics, 19, 46-70.

Bagdonavičius V and Nikulin M (2011), Chi-squared goodness-of-fit test for right censored data, International Journal of Applied Mathematics and Statistics, 24, 30-50.

Bagdonavičius V, Levuliene RJ, and Nikulin M (2013). Chi-squared goodness-of-fit tests for parametric accelerated failure time models, Communications in Statistics- Theory and Methods, 42, $2768-2785$.

Bjerkedal T (1960). Acquisition of resistance in Guinea pigs infected with different doses of virulent tubercle bacilli, American Journal of Hygiene, 72, 130-148.

Burr IW (1942). Cumulative frequency functions, Annals of Mathematical Statistics, 13, 215-232.

Burr IW (1968). On a general system of distributions III: The simplerange, Journal of the American Statistical Association, 63, 636-643.

Burr IW (1973). Parameters fora general system of distributions to match a grid of 3 and 4, Сотmunications in Statistics, 2, 1-21.

Elsayed HA and Yousof HM (2019). A new Lomax distribution for modeling survival times and taxes revenue data sets, Journal of Statistics and Applications, 2, 35-58.

Elsayed HAH and Yousof HM (2021). Extended poisson generalized Burr XII distribution, Journal of Applied Probability and Statistics, forthcoming,

Gad AM, Hamedani GG, Salehabadi SM, and Yousof HM (2019). The Burr XII-Burr XII distribution: mathematical properties and characterizations, Pakistan Journal of Statistics, 35, 229-248.

Gijbels I and Gurler U (2003). Estimation of a change-point in a hazard function based on censored data, Lifetime Data Analysis, 9, 395-411.

Goual H, Yousof HM, and Ali MM (2019). Validation of the odd Lindley exponentiated exponential by a modified goodness of fit test with applications to censored and complete data, Pakistan Journal of Statistics and Operation Research, 15, 745-771.

Goual H and Yousof HM (2020). Validation of Burr XII inverse Rayleigh model via a modified chi-squared goodness-of-fit test, Journal of Applied Statistics, 47, 393-423.

Ibrahim M, Altun E, Goual H, and Yousof HM (2020). Modified goodness-of-fit type test for cen- 
sored validation under a new Burr type XII distribution with different methods of estimation and regression modeling, Eurasian Bulletin of Mathematics, 3, 162-182.

Ibrahim M, Yadav AS, Yousof HM, Goual H, and Hamedani GG (2019). A new extension of Lindley distribution: modified validation test, characterizations and different methods of estimation, Communications for Statistical Applications and Methods, 26, 473-495.

Mansour MM, Ibrahim M, Aidi K, Shafique Butt N, Ali MM, Yousof HM, and Hamed MS (2020a). A new log-logistic lifetime model with mathematical properties, copula, modified goodness-of-fit test for validation and real data modeling, Mathematics, 8, 1508.

Mansour M, Rasekhi M, Ibrahim M, Aidi K, Yousof HM, and Elrazik EA (2020b). A new parametric life distribution with modified Bagdonavičius-Nikulin goodness-of-fit test for censored validation, properties, applications, and different estimation methods, Entropy, 22, 592.

Nichols MD and Padgett WJ (2006). A bootstrap control chart for Weibull percentiles, Quality and Reliability Engineering International, 22, 141-151.

Rodriguez RN (1977). A guide to the Burr type XII distributions, Biometrika, 64, 129-134.

Salah MM, El-Morshedy M, Eliwa MS, and Yousof HM (2020). Expanded Fréchet model: Mathematical properties, copula, different estimation methods, applications and validation testing, Mathematics, 8.

Tadikamalla PR (1980). A look at the Burr and related distributions, International Statistical Review, 48, 337-344.

Voinov V, Nikulin M, and Balakrishnan N (2013). Chi-Squared Goodness of Fit Tests with Applications, Academic Press, Elsevier.

Yadav AS, Goual H, Alotaibi RM, Ali MM, and Yousof HM (2020). Validation of the Topp-LeoneLomax model via a modified Nikulin-Rao-Robson goodness-of-fit test with different methods of estimation, Symmetry, 12.

Yousof HM, Aidi K, Hamedani GG, and Ibrahim M (2021). A new parametric lifetime distribution with modified Chi-square type test for right censored validation, characterizations and different estimation methods, Pakistan Journal of Statistics and Operation Research, 17.

Yousof HM, Altun E, Ramires TG, Alizadeh M, and Rasekhi M (2018). A new family of distributions with properties, regression models and applications, Journal of Statistics and Management Systems, 21, 163-188.

Yousof HM, Altun E, Rasekhi M, Alizadeh M, Hamedani GG, and Ali MM (2019). A new lifetime model with regression models, characterizations and applications, Communications in StatisticsSimulation and Computation, 48, 264-286. 
